\title{
HYDRODYNAMIC MODELS OF SELF-ORGANIZED DYNAMICS: DERIVATION AND EXISTENCE THEORY*
}

\author{
PIERRE DEGOND ${ }^{\dagger}$, JIAN-GUO LIU ${ }^{\ddagger}$, SEBASTIEN MOTSCH ${ }^{\S}$, AND \\ VLADISLAV PANFEROV
}

\begin{abstract}
This paper is concerned with the derivation and analysis of hydrodynamic models for systems of self-propelled particles subject to alignment interaction and attraction-repulsion. Introducing various scalings, the effects of the alignment and attraction-repulsion interactions give rise to a variety of hydrodynamic limits. For instance, local alignment produces a pressure term at the hydrodynamic limit whereas near-local alignment induces a viscosity term. Depending on the scalings, attraction-repulsion either yields an additional pressure term or a capillary force (also termed 'Korteweg force'). The hydrodynamic limits are shown to be symmetrizable hyperbolic systems with viscosity terms. A local-in-time existence result is proved in the $2 \mathrm{D}$ case for the viscous model and in the 3D case for the inviscid model.
\end{abstract}

Key words. Self-propelled particles, alignment dynamics, hydrodynamic limit, diffusion correction, weakly non-local interaction, symmetrizable hyperbolic system, energy method, local wellposedness, capillary force, Korteweg force, attraction-repulsion potential.

AMS subject classifications. 35L60, 35K55, 35Q80, 82C05, 82C22, 82C70, 92D50.

1. Introduction. There has been an intense literature about the modeling of interactions between individuals among animal societies such as fish schools, bird flocks, herds of mammals, etc. We refer e.g. to $[1,2,35,9,21]$ but an exhaustive bibliography is out of reach. Among these models, the Vicsek model [40] has received particular attention due to its simplicity and the universality of its qualitative features. This model is a discrete particle model (or 'Individual-Based Model' or 'Agent-Based model') which consists of a time-discretized set of Ordinary Differential Equations for the particle positions and velocities. The velocities are supposed to be of constant norm and are updated according to an alignment rule: each agent tries to align its velocity to that of its neighbors in some sensing region. Some angular noise is added to account for stochastic fluctuations. A time-continuous version of this model and its kinetic formulation are available in $[10,13]$. A rigorous derivation of this kinetic model from the time-continuous Vicsek model can be found in [3].

The present work starts with an Individual-Based Model which appears to be a generalization of the Vicsek model [40] with the addition of an attraction-repulsion interaction potential (see e.g. [7, 10,22]). Again, the self-propulsion speed is supposed to be constant and identical for all the particles. Therefore, the velocity variable reduces to its orientation. In addition to the alignment rule which tends to relax the particle velocities to their local orientation (already present in the original Vicsek model [40]), the particle interactions involve an attraction-repulsion rule. It makes

\footnotetext{
*Received July 12, 2012; accepted for publication December 21, 2012.

†Université de Toulouse, UPS, INSA, UT1, UTM, Institut de Mathématiques de Toulouse, F31062 Toulouse, France; 2-CNRS; Institut de Mathématiques de Toulouse UMR 5219, F-31062 Toulouse, France (pierre.degond@math.univ-toulouse.fr).

${ }^{\ddagger}$ Department of Physics and Department of Mathematics, Duke University, Durham, NC 27708, USA (jliu@phy.duke.edu).

${ }^{\S}$ Center of Scientific Computation and Mathematical Modeling (CSCAMM), University of Maryland, College Park, MD 20742, USA (smotsch@cscamm.umd.edu).

IDepartment of Mathematics, California State University, Northridge, 18111 Nordhoff St., Northridge, CA 91330-8313, USA (vladislav.panferov@csun.edu).
} 
the particles move closer or farther away from each other according to whether the interaction is attractive or repulsive. Typically, we will consider short-range attraction and long-range repulsion, in the spirit of the three-zone model [2, 35, 9]. Additionally, velocity orientations undergo a Brownian motion which describes the effect of stochastic fluctuations. Therefore, this model can be seen as a continuous version of the three zone model $[2,35,9]$.

In [13], the hydrodynamic limit of the time-continuous Vicsek model is performed through an asymptotic analysis of its kinetic version [3]. The resulting model is a system of balance equations for the density and mean velocity orientation (or polarization vector). The model has been later referred to as the Self-Organized Hydrodynamics $(\mathrm{SOH})$ and is studied numerically in $[12,29]$. The main concern of the paper is to study the modifications of the $\mathrm{SOH}$ model that are induced by (i) the introduction of the attraction-repulsion force as described above and (ii) different scaling assumptions about the size of the sensing region which involve a higher level of nonlocality. As proved in [13], the strict combination of alignment and noise results in the appearance of a pressure term in the $\mathrm{SOH}$ model. Introducing an attraction-repulsion force and spanning various scaling assumptions on this force and on the size of the sensing region, we obtain a variety of effects that are not encompassed in [13]. First, we try to take a better account of the non-local character of the interaction forces than in [13]. To this aim, the scaling assumption relating the range of the interaction force to the micro and macro-scopic scales is modified. The chosen scaling makes the interaction range large compared to the microscopic scale but small compared to the macroscopic one. The non-local character of the interaction force at the microscopic level results in the appearance of higher order derivative terms, such as viscosity terms, at the macroscopic level. The specific effect of the attraction-repulsion force in the hydrodynamic limit is to add either an extra pressure term or a capillary (or Korteweg) force term depending on the chosen scalings.

Beyond the statements of the models, we prove a local-in-time existence theorem in the $2 \mathrm{D}$ case for the viscous model (when the non-local effects are retained) and in the $3 \mathrm{D}$ case for the inviscid model (when the non-local effects are omitted). Both proofs rely on a suitable symmetrization of the system and on the energy method.

Hydrodynamic models are attractive over particle ones due to their computational efficiency. For this reason, many such models have been proposed in the literature $[5,6,8,18,27,28,37,38]$. However, most of them are phenomenological. [13] proposes one of the first rigorous derivations of a hydrodynamic version of the Vicsek model (see also [24, 32, 33] for phenomenological derivations). It has been expanded in [14] to account for a model of fish behavior where particles interact through curvature control, and in [15] to include diffusive corrections. Other variants have also been investigated. For instance, [19] studies the influence of a vision angle and of the dependency of the alignment frequency upon the local density. [11, 20] propose a modification of the model which results in phase transitions from disordered to ordered equilibria as the density increases and reaches a threshold, in a way similar to polymer models $[17,30]$.

The organization of the paper is as follows. In Section 2, we introduce the model of self-propelled particles and set up the associated kinetic equation. We then discuss various scalings which lead to the derivation of the studied hydrodynamic models. We introduce four dimensionless parameters in the problem: the scaled interaction meanfree path $\varepsilon$, the radius of the sensing region $\eta$, the noise intensity $\delta$ and the relative strength between the attraction-repulsion and the alignment forces $\tilde{\chi}$. The scaling considered in [13] ignores the attraction-repulsion force (i.e. $\tilde{\chi}=0$ ) and supposes 
that $\varepsilon=\eta \rightarrow 0$ with $\delta=\mathcal{O}(1)$. Here, we investigate four different scaling relations.

1. The weakly non-local interaction scaling without noise: $\eta=\sqrt{\varepsilon}, \delta=0$, $\tilde{\chi}=\eta^{2}$. The resulting model is a viscous version of the $\mathrm{SOH}$ model. We recall (see e.g. [13]) that the $\mathrm{SOH}$ model is a variant of the inviscid isothermal compressible Euler equations where the fluid velocity is constrained to be of unit norm. More precisely, in the SOH model, the unknown fluid velocity is replaced by the fluid velocity direction (or polarization vector). As a result of this constraint, the momentum balance equation is non-conservative. In this scaling, we assume that the solutions of the kinetic equation are monokinetic. We justify this assumption by studying the space homogeneous kinetic model and prove that the solutions converge on the fast $\varepsilon$ time scale to the monokinetic distribution. We also highlight the variational structure of this space homogeneous kinetic model. Note that the scaling assumption $\eta=\sqrt{\varepsilon}$ is different from the one used in [13]. It corresponds to increasing the size of the interaction region in the microscopic variables by a factor $1 / \sqrt{\varepsilon}$, as $\varepsilon \rightarrow 0$. Therefore, more and more non-local effects are picked up in the hydrodynamic limit. These non-local effects give rise to the viscosity term in the viscous $\mathrm{SOH}$ model, which makes an original addition from previous works.

2. The local interaction scaling with noise. This is the scaling proposed in [13]. It is recalled here just for the sake of comparisons. It consists in letting $\eta \ll \varepsilon, \delta=\mathcal{O}(1), \tilde{\chi} \leq \eta^{2}$. The resulting model is the inviscid SOH model (see presentation in the previous item).

3. The weakly non-local interaction scaling with noise. This scaling unifies the two previous scalings. It consists in letting $\eta=\sqrt{\varepsilon}, \delta=\mathcal{O}(1), \tilde{\chi}=\eta^{2}$. Again, the resulting model is a viscous $\mathrm{SOH}$ model, but with modified coefficients as compared to the first scaling. We note however, that in the zero noise limit $\delta \rightarrow 0$, we recover the system obtained with the first scaling, which provides another justification of the monokinetic assumption in the derivation of the model.

4. Strong potential force scaling. This corresponds to $\eta=\sqrt{\varepsilon}, \delta=\mathcal{O}(1), \tilde{\chi}=1$. Therefore, here, the attraction repulsion force is of the same order as the alignment force. However, we make the additional assumption that the zeroth order moment of the potential is zero, which expresses some kind of balance between the attraction and repulsion effects. Equivalently, we can say that we modify the potential with van der Waals modification [39]. This results in an $\mathrm{SOH}$ model with the addition of a term analog to the capillary force (or Korteweg term [23]), induced from the attractive part of the potential.

In Section 3, we prove local well-posedness for all the models derived in Section 2, except the last one (strong potential force scaling). All these systems have the same form of a symmetrizable hyperbolic system with additional viscosity. In Section 3.1, we prove the local-in-time existence of solutions for the viscous system in $2 \mathrm{D}$ and in Section 3.2, we show the same result for the inviscid system in 3D based on the energy method. Finally, a conclusion is drawn in Section 4.

Acknowledgements. The authors wish to acknowledge the hospitality of Mathematical Sciences Center and Mathematics Department of Tsinghua University where this research was performed. The research of J.-G. L. was partially supported by NSF grant DMS 10-11738. This work has been partially supported by KI-Net NSF RNMS grant No. 1107291. 


\section{Derivation of self-organized hydrodynamic models.}

2.1. Individual-based model of self-alignment with attractionrepulsion. The starting point of this study is an Individual-Based Model of particles interacting through self-alignment [40] and attraction-repulsion $[2,35,9]$. Specifically, we consider $N$ particles $x_{k} \in \mathbb{R}^{n}, k=1, \ldots, N$, moving at a constant speed $a>0$ with direction $v_{k} \in \mathbb{S}^{n-1}$. Each particle adjusts its velocity to align with its neighbors or to get closer to or further away from them. The evolution of each particle is modeled by the following dynamics:

$$
\begin{aligned}
& \frac{d x_{k}}{d t}=a v_{k}, \\
& d v_{k}=P_{v_{k}^{\perp}} \circ\left(\sigma \bar{v}_{k} d t+\sqrt{2 d} d B_{t}^{k}\right) .
\end{aligned}
$$

Here, $P_{v_{k}^{\perp}}=\mathrm{Id}-v_{k} \otimes v_{k}$ is the projection matrix onto the normal plane to $v_{k}$. It ensures that $v_{k}$ stays of norm 1 . $B_{t}^{k}$ are independent Brownian motions in $\mathbb{R}^{n}$ and the stochastic differential equation (2.2) is to be understood in the Stratonovitch sense. The quantities $d$ and $\sigma$ represent the noise and social force intensities respectively. The social force describes attraction at large distances, repulsion at short distances and alignment in the intermediate range, in accordance to the so-called three zone model $[2,35,9]$. Both the alignment and attraction-repulsion rules are encoded in the vector $\bar{v}_{k}$ :

$$
\bar{v}_{k}=\frac{j_{k}+\chi r_{k}}{\left|j_{k}+\chi r_{k}\right|}
$$

where $j_{k}$ counts for the alignment and $r_{k}$ for the attraction-repulsion:

$$
\begin{aligned}
& j_{k}=\frac{1}{N} \sum_{j=1}^{N} K\left(\left|x_{j}-x_{k}\right|\right) v_{j}, \\
& r_{k}=-\left.\nabla_{x}\left(\frac{1}{N} \sum_{j=1}^{N} \Phi\left(\left|x-x_{j}\right|\right)\right)\right|_{x=x_{k}}=-\frac{1}{N} \sum_{j=1}^{N} \Phi^{\prime}\left(\left|x_{k}-x_{j}\right|\right) \frac{x_{k}-x_{j}}{\left|x_{k}-x_{j}\right|},
\end{aligned}
$$

and $\chi$ measures the strength of the attraction-repulsion force relative to the alignment force (and has physical dimension of a length). We denote by $\Phi^{\prime}$ the derivative of $\Phi$ with respect to $|x|$. We assume that the kernel $K$ is positive and that both kernels $K$ and $\Phi$ are integrable and that $K$ and $\Phi$ are 'normalized' in the following sense:

$$
K \geq 0, \quad \int_{\mathbb{R}^{n}} K(|x|) d x=1, \quad \int_{\mathbb{R}^{n}}|\Phi(|x|)| d x=1 .
$$

In addition, we assume that $\Phi$ tends to zero at infinity (i.e. $\Phi(r) \rightarrow 0$ when $r \rightarrow \infty$ ). We note that $\Phi^{\prime}$ can be negative in some regions (the repulsion regions) and positive in other regions (the attraction regions). In figure 1, we give examples of functions $K$ and $\Phi$ modeling the popular three-zone model for fish behavior $[2,9,31]$. We make the assumption that both potentials describe a certain interaction scale $R$ and we highlight this fact by writing

$$
K(|x|)=\frac{1}{R^{n}} \tilde{K}\left(\frac{|x|}{R}\right), \quad \Phi(|x|)=\frac{1}{R^{n}} \tilde{\Phi}\left(\frac{|x|}{R}\right),
$$

where $\tilde{K}$ and $\tilde{\Phi}$ are reference interaction kernels and $R$ is the sensing radius. 




FIG. 1. The three-zone model (repulsion-alignment-attraction) can be viewed as a special choice of kernel $K$ and potential $\Phi$.

When the number of particles becomes large (i.e. $N \rightarrow \infty$ ), one can formally derive the equation satisfied by the particle distribution function $f(x, v, t)$ (i.e. the probability distribution of the particles in phase-space $(x, v))$. Under suitable assumptions $[3,13,36], f$ satisfies the following kinetic equation:

$$
f_{t}+a v \cdot \nabla_{x} f=-\sigma \nabla_{v} \cdot\left[\left(P_{v} \perp v_{f}\right) f\right]+d \Delta_{v} f,
$$

where

$$
\begin{aligned}
& v_{f}=\frac{j_{f}+\chi r_{f}}{\left|j_{f}+\chi r_{f}\right|}, \\
& j_{f}=\frac{1}{R^{n}} \int_{x^{\prime}, v^{\prime}} \tilde{K}\left(\frac{\left|x^{\prime}-x\right|}{R}\right) v^{\prime} f\left(x^{\prime}, v^{\prime}, t\right) d x^{\prime} d v^{\prime}, \\
& r_{f}=-\frac{1}{R^{n}} \nabla_{x} \int_{x^{\prime}, v^{\prime}} \tilde{\Phi}\left(\frac{\left|x^{\prime}-x\right|}{R}\right) f\left(x^{\prime}, v^{\prime}, t\right) d x^{\prime} d v^{\prime},
\end{aligned}
$$

The operator $\nabla_{v}$ denotes the tangential gradient on the sphere and $\Delta_{v}$ is the LaplaceBeltrami operator. Using this kinetic formulation, we want to explore the asymptotic behavior of the model in different regimes. For this purpose, we introduce a scaling of the physical variables. This is the purpose of the next section.

2.2. Scaling parameters. We now introduce dimensionless variables and scaling assumptions. Let $t_{0}$ be a time unit and let $x_{0}=a t_{0}, f_{0}=1 /\left(x_{0}\right)^{n}$. We choose $t_{0}$ in such a way that $\sigma t_{0}=1$ and introduce the quantity $\delta=d t_{0}=\mathcal{O}(1)$. Introducing 
new variables $\tilde{x}=x / x_{0}, \tilde{t}=t / t_{0}, \tilde{f}(\tilde{x}, v, \tilde{t})=f(x, v, t) / f_{0}$, eq. (2.6) is written:

$$
\tilde{f}_{\tilde{t}}+v \cdot \nabla_{\tilde{x}} \tilde{f}=-\nabla_{v} \cdot\left[\left(P_{v \perp} v_{\tilde{f}}\right) \tilde{f}\right]+\delta \Delta_{v} \tilde{f} .
$$

In this choice of units, the influence of the social force and of the noise are of order unity. We now introduce

$$
\tilde{R}=\frac{R}{x_{0}}, \quad \tilde{\chi}=\frac{\chi}{x_{0}}
$$

Then, eqs (2.7)-(2.9) are now written in dimensionless variables:

$$
\begin{aligned}
v_{\tilde{f}} & =\frac{\tilde{j}_{\tilde{f}}+\tilde{\chi} \tilde{r}_{\tilde{f}}}{\left|\tilde{j}_{\tilde{f}}+\tilde{\chi} \tilde{r}_{\tilde{f}}\right|}, \\
\tilde{j}_{\tilde{f}} & =\int_{\tilde{x}^{\prime}, v^{\prime}} \tilde{K}\left(\frac{\left|\tilde{x}^{\prime}-\tilde{x}\right|}{\tilde{R}}\right) v^{\prime} \tilde{f}\left(\tilde{x}^{\prime}, v^{\prime}, \tilde{t}\right) d \tilde{x}^{\prime} d v^{\prime}, \\
\tilde{r}_{\tilde{f}} & =-\nabla_{\tilde{x}} \int_{\tilde{x}^{\prime}, v^{\prime}} \tilde{\Phi}\left(\frac{\left|\tilde{x}^{\prime}-\tilde{x}\right|}{\tilde{R}}\right) \tilde{f}\left(\tilde{x}^{\prime}, v^{\prime}, \tilde{t}\right) d \tilde{x}^{\prime} d v^{\prime},
\end{aligned}
$$

where $\tilde{j}_{\tilde{f}}(\tilde{x}, \tilde{t})=R^{n} j_{f}(x, t)$ and $\tilde{r}_{\tilde{f}}(\tilde{x}, \tilde{t})=R^{n} r_{f}(x, t)$.

We now introduce hydrodynamic scale. This means that we change the space and time units to new ones $x_{0}^{\prime}, t_{0}^{\prime}$ which are large compared to the microscopic units $x_{0}$, $t_{0}$. Specifically, we let $\varepsilon \ll 1$ be a small parameter and define $x_{0}^{\prime}=x_{0} / \varepsilon, t_{0}^{\prime}=t_{0} / \varepsilon$. By doing so, we change the space and time variables $\tilde{x}$ and $\tilde{t}$ to macroscopic variables $\hat{x}=\varepsilon \tilde{x}, \hat{t}=\varepsilon \tilde{t}$ and define $\hat{f}(\hat{x}, v, \hat{t})=\varepsilon^{-n} \tilde{f}(\tilde{x}, v, \tilde{t})$. Finally, we define

$$
\eta=\varepsilon \tilde{R}
$$

In all sections but Section 2.6, we scale the relative intensity of the attraction-repulsion force to the alignment force $\tilde{\chi}$ as:

$$
\tilde{\chi}=\eta^{2}
$$

We refer to this scaling as the weak potential force scaling. In Section 2.6, this assumption will be changed to $\tilde{\chi}=1$ under van der Waals modification of the potential. This other scaling is referred to as the strong potential force scaling.

Performing this new change of variables and dropping the hats on the variables for the sake of simplicity, we are led to the following system for the kinetic distribution function $f(x, v, t)$ :

$$
f_{t}+v \cdot \nabla_{x} f=\frac{1}{\varepsilon}\left(-\nabla_{v} \cdot\left[\left(P_{v} v_{f}^{\eta}\right) f\right]+\delta \Delta_{v} f\right),
$$

where, in the weak potential force scaling (2.14), we have:

$$
\begin{aligned}
v_{f}^{\eta} & =\frac{j_{f}^{\eta}+\eta^{2} r_{f}^{\eta}}{\left|j_{f}^{\eta}+\eta^{2} r_{f}^{\eta}\right|}, \\
j_{f}^{\eta} & =\int_{\left(x^{\prime}, v^{\prime}\right) \in \mathbb{R}^{n} \times \mathbb{S}^{n-1}} K\left(\frac{\left|x^{\prime}-x\right|}{\eta}\right) v^{\prime} f\left(x^{\prime}, v^{\prime}, t\right) d x^{\prime} d v^{\prime}, \\
r_{f}^{\eta} & =-\nabla_{x} \int_{\left(x^{\prime}, v^{\prime}\right) \in \mathbb{R}^{n} \times \mathbb{S}^{n-1}} \Phi\left(\frac{\left|x^{\prime}-x\right|}{\eta}\right) f\left(x^{\prime}, v^{\prime}, t\right) d x^{\prime} d v^{\prime} .
\end{aligned}
$$


Reminding that $K$ is normalized by $(2.5)$, we denote:

$$
k=\frac{1}{2 n} \int_{\xi \in \mathbb{R}^{n}} K(|\xi|)|\xi|^{2} d \xi, \quad \Theta=\int_{\xi \in \mathbb{R}^{n}} \Phi(|\xi|) d \xi .
$$

The potential $\Phi$ is said to be globally repulsive if $\Theta \geq 0$. Indeed, we will see that the introduction of the potential gives rise to an additional isothermal pressure force associated to an equivalent temperature $\Theta$ in the $\mathrm{SOH}$ model. If $\Theta$ is negative, then the $\mathrm{SOH}$ model may become nonhyperbolic. This occurs when particle attraction at large distances overtakes particle repulsion at short distances. Such potentials are thus referred to as globally attractive ones. We want to avoid them. Therefore, we make the assumption of a globally repulsive potential, with $\Theta \geq 0$.

Defining the moments $\rho_{f}$ and $\rho_{f} u_{f}$ of $f$ by

$$
\rho_{f}=\int_{v \in \mathbb{S}^{n-1}} f(v) d v, \quad \rho_{f} u_{f}=\int_{v \in \mathbb{S}^{n-1}} f(v) v d v
$$

we have the following Taylor expansion of the quantity $v_{f}^{\eta}$ (given by $(2.16)$ ) in the weak potential force scaling:

$$
\begin{aligned}
& v_{f}^{\eta}=\Omega_{f}+\eta^{2} \frac{1}{\rho_{f}\left|u_{f}\right|} \ell_{f}+o\left(\eta^{2}\right), \\
& \Omega_{f}=\frac{u_{f}}{\left|u_{f}\right|}, \quad \ell_{f}=P_{\Omega_{f}^{\perp}}\left(k \Delta_{x}\left(\rho_{f} u_{f}\right)-\Theta \nabla_{x} \rho_{f}\right) .
\end{aligned}
$$

Inserting this expression into the kinetic equation (2.15), we get

$$
\begin{aligned}
f_{t}+v \cdot \nabla_{x} f & =-\frac{1}{\varepsilon} \nabla_{v} \cdot\left[\left(P_{v \perp} \Omega_{f}\right) f\right] \\
& -\frac{\eta^{2}}{\varepsilon} \frac{1}{\rho_{f}\left|u_{f}\right|} \nabla_{v} \cdot\left[\left(P_{v^{\perp}} \ell_{f}\right) f\right]+\frac{\delta}{\varepsilon} \Delta_{v} f+o\left(\frac{\eta^{2}}{\varepsilon}\right) .
\end{aligned}
$$

We now consider four different scaling limits which lead to models for which we will prove local existence of classical solutions. They are all concerned with the limits $\eta \rightarrow 0$ and $\varepsilon \rightarrow 0$ but assume different relations between $\eta$ and $\varepsilon$. In addition, in some cases, we distinguish between the noisy case $\delta \neq 0$ and the noiseless case $\delta=0$. The first three scalings are relative to the weak potential force scaling, while the fourth one considers the strong potential force scaling. Specifically, these scalings are (see the discussion at the end of Section 1):

1. Weakly non-local interaction without noise: $\eta^{2} / \varepsilon=\mathcal{O}(1)$ and $\delta=0$.

2. Local interaction with noise: $\eta^{2} / \varepsilon=o(1)$ and $\delta=\mathcal{O}(1)$.

3. Weakly non-local interaction with noise: $\eta^{2} / \varepsilon=\mathcal{O}(1)$ and $\delta=\mathcal{O}(1)$

4. Strong potential force scaling: large attraction-repulsion force $\tilde{\chi}=\mathcal{O}(1)$, with van der Waals modification of the potential. Note that in this last scaling, expansion (2.19) is not true (and consequently neither is (2.20)). The correct expansion for this case will be performed in Section 2.6.

The weakly non-local interaction scaling allows us to retain some of the nonlocality of the social force in the macroscopic model, while the local one does not. Indeed, $\varepsilon$ corresponds to the characteristic distance needed by an individual to react to the social force, while $\eta$ is the typical distance at which agents are able to detect their congeners. For instance, the local scaling is satisfied if $\eta=O(\varepsilon)$. In this case, these two distances are of the same order of magnitude. By contrast, in the non-local 
interaction scaling, the agents' detection region is large compared to the reaction distance. Which one of these two regimes is biologically relevant depends on the situation. For instance, we can imagine that the local interaction scaling will be more relevant in denser swarms because in such systems, far agents are concealed by closer ones. We will present these various scaling sequentially, starting from the first one (Section 2.3), then moving successively towards the second (Section 2.4), third (Section 2.5) and fourth (Section 2.6) ones.

2.3. Weakly non-local interaction scaling without noise. In this scaling limit, we assume no noise $\delta=0$ and the following ordering between the two parameters $\varepsilon$ and $\eta$ :

$$
\varepsilon \rightarrow 0, \quad \eta \rightarrow 0, \quad \frac{\eta^{2}}{\varepsilon} \rightarrow 1
$$

$f^{\varepsilon}$ satisfies (keeping only the $\mathcal{O}(1)$ terms in $\varepsilon$ or larger):

$$
f_{t}^{\varepsilon}+v \cdot \nabla_{x} f^{\varepsilon}+\frac{1}{\rho_{f^{\varepsilon}}\left|u_{f^{\varepsilon}}\right|} \nabla_{v} \cdot\left[\left(P_{v^{\perp}} \ell_{f^{\varepsilon}}\right) f^{\varepsilon}\right]=-\frac{1}{\varepsilon} \nabla_{v} \cdot\left[\left(P_{v^{\perp}} \Omega_{f^{\varepsilon}}\right) f^{\varepsilon}\right] .
$$

This equation results from (2.20) by making $\eta^{2} / \varepsilon=1$ in the second term at the righthand side of (2.20) and moving it to the left-hand side and by omitting the third term.

In order to study the limit $\varepsilon \rightarrow 0$, we have to determine the stable equilibria i.e. the stable solutions of $\nabla_{v} \cdot\left[\left(P_{v^{\perp}} \Omega_{f^{\varepsilon}}\right) f^{\varepsilon}\right]=0$. Such stable equilibria are defined as the limits as time tends to infinity of the spatially homogeneous equation:

$$
f_{t}=-\frac{1}{\varepsilon} \nabla_{v} \cdot\left[\left(P_{v^{\perp}} \Omega_{f}\right) f\right] .
$$

In the following lemma, we show that its solution relaxes to a monokinetic distribution:

$$
f^{\varepsilon}(v, t) \rightarrow \rho \delta_{\Omega}(v),
$$

with $\rho>0, \Omega \in \mathbb{S}^{n-1}$ and where $\delta_{\Omega}(v)$ denotes the Dirac delta at $v=\Omega$. Moreover, this limit occurs at the fast $\varepsilon$ time scale. More precisely, we have

Proposition 2.1. We assume that $\left.\int f\right|_{t=0} d v=1$ so that $\int f(v, t) d v=1$ and $u=\int v f(v) d v$ for all times (we omit the index $f$ when the context is clear). We also assume that $u_{t=0} \neq 0$, otherwise, the dynamics is not defined. Then, any stable limit point of the solution $f(t)$ of (2.22) as $t \rightarrow \infty$ is of the form $\delta_{\Omega}(v)$ for some $\Omega \in \mathbb{S}^{n-1}$.

Proof. We introduce the free energy:

$$
\mathcal{F}(f)=\int(1-u \cdot v) f(v) d v
$$

and note that $\mathcal{F}(f)=1-|u|^{2} \geq 0$ because $|u| \leq 1$. We note that $|u|^{2}$ is the classical order parameter [40]. In Lemma 2.2 below, we prove that $\mathcal{F}(f)$ satisfies the following dissipation equation:

$$
\frac{\partial}{\partial t} \mathcal{F}(f)+\mathcal{D}(f)=0, \quad \mathcal{D}(f)=\frac{2}{\varepsilon} \int \frac{|u|^{2}-(v \cdot u)^{2}}{|u|} f(v) d v .
$$


Since $|u|^{2}-(v \cdot u)^{2} \geq 0$, we have

$$
\frac{\partial}{\partial t} \mathcal{F}(f) \leq 0
$$

Therefore, $\mathcal{F}(f)$ is a decreasing function of time. Furthermore, if $f$ is a distribution such that

$$
\mathcal{D}(f)=0=\frac{2}{\varepsilon} \int \frac{|u|^{2}-(v \cdot u)^{2}}{|u|} f(v) d v=\frac{|u|}{2 \varepsilon} \int\left|v-\frac{u}{|u|}\right|^{2}\left|v+\frac{u}{|u|}\right|^{2} f(v) d v,
$$

then $f$ is of the form of a dipole:

$$
f=\alpha \delta_{\Omega}(v)+(1-\alpha) \delta_{-\Omega}(v)
$$

where $\alpha \in[0,1]$ and $\Omega=\frac{u}{|u|} \in \mathbb{S}^{n-1}$. Indeed, $f$ is a positive measure and the quantity at the right-hand side of $(2.25)$ can only be zero if $f$ is supported by either $\Omega$ or $-\Omega$. Now, any dipole such that $\alpha \neq 1$ is trajectorily unstable. Indeed, eq. (2.22) is a first order conservation law on the sphere. The characteristics of this equation are given by:

$$
\dot{v}=\frac{1}{\varepsilon} P_{v^{\perp}} \Omega .
$$

This dynamical system has two stationary points: $\Omega$ and $-\Omega$. The point $\Omega$ is a sink and is stable. By contrast, the point $-\Omega$ is a source and hence is unstable. Therefore, a dipole with $\alpha \neq 1$ can never be reached in the course of the dynamics, unless it is initially a dipole or a perturbation of a dipole with cylindrical symmetry around the dipole axis. We illustrate the geometry of the characteristics given by (2.27) in Fig. 2 , which shows the source $-\Omega$ and sink $\Omega$ of this system.

To conclude, we collect all these observations. The quantity $\mathcal{F}(f)$ is decreasing with time and tends to a limit $\mathcal{F}_{\infty}$ as $t \rightarrow \infty$. Therefore, the dissipation rate $\mathcal{D}(f)$ tends to 0 . This implies that any limit point $f_{\infty}$ of $f(t)$ satisfies $\mathcal{D}\left(f_{\infty}\right)=0$. Owing to the instability of the dipole, this implies that any stable limit point $f_{\infty}$ is of the form $f_{\infty}=\delta_{\Omega}(v)$ for some $\Omega \in \mathbb{S}^{n-1}$ with typical convergence rate $\varepsilon^{-1}$.

LEMMA 2.2. Any solution $f$ of (2.22) satisfies (2.24).

Proof. We write

$$
\begin{aligned}
\frac{\partial}{\partial t} \mathcal{F}(f) & =\int(1-(u \cdot v)) \partial_{t} f(v) d v-u_{t} \cdot \int v f(v) d v \\
& =\int(1-(u \cdot v)) \partial_{t} f(v) d v-\int v \partial_{t} f(v) d v \cdot u \\
& =\partial_{t}\left(\int f(v) d v\right)-2 \int(u \cdot v) \partial_{t} f(v) d v \\
& =-2 \int(u \cdot v) \partial_{t} f(v) d v
\end{aligned}
$$

To pass from the first to the second line in the last term, we note that $\int v f(v) d v=u$ and therefore, $u_{t} \cdot \int v f(v) d v=u \cdot \int v f_{t}(v) d v$. In the last equality, we have used that $\int f(v, t) d v=1$. Now, multiplying $(2.22)$ by $-2(u \cdot v)$, integrating with respect to $v$, using Green's formula on the sphere and the fact that $\nabla_{v}(v \cdot u)=P_{v \perp} u$, we get:

$$
\frac{\partial}{\partial t} \mathcal{F}(f)+\frac{2}{\varepsilon} \int\left(P_{v^{\perp}} u \cdot P_{v^{\perp}} \Omega\right) f(v) d v=0 .
$$






FIG. 2. The trajectories of the differential equation (2.27) on the sphere, showing the source $-\Omega$ and $\operatorname{sink} \Omega$.

But by definition of $\Omega$, we have $\Omega=\frac{u}{|u|}$, so that we find:

$$
P_{v^{\perp}} u \cdot P_{v^{\perp}} \Omega=\frac{1}{|u|}\left(|u|^{2}-(u \cdot v)^{2}\right),
$$

which leads to the result.

Now, we return to the space-inhomogeneous system (2.21). Using the previous study, we can state the

THEOREM 2.3. If $\varepsilon$ tends to 0 in (2.21), we formally have $f^{\varepsilon} \rightarrow \rho \delta_{\Omega}$ where $\delta_{\Omega}(v)$ is the Dirac delta distribution at $v=\Omega$ and $\rho=\rho(x, t)$ and $\Omega=\Omega(x, t)$ satisfy the following SOH system:

$$
\begin{aligned}
& \partial_{t} \rho+\nabla_{x} \cdot(\rho \Omega)=0, \\
& \partial_{t}(\rho \Omega)+\nabla_{x} \cdot(\rho \Omega \otimes \Omega)+\Theta P_{\Omega^{\perp}} \nabla_{x} \rho=k P_{u^{\perp}} \Delta_{x}(\rho \Omega) .
\end{aligned}
$$

We formally have $|\Omega(\cdot, t)|=1$ for all $t>0$ as soon as the initial data $\left(\rho_{0}, \Omega_{0}\right)$ satisfies $\left|\Omega_{0}\right|=1$.

REMARK 2.1. The repulsive force contributes for a pressure term at the left-hand side of the momentum equation, which otherwise would not be strictly hyperbolic, and would fall in the class of Pressureless Gas Dynamics models [4].

Elements of the proof. We begin with some remarks. For the sake of simplicity, we note

$$
\langle\varphi\rangle_{\mathbb{S}^{n-1}}=\int_{v \in \mathbb{S}^{n-1}} \varphi(v) d v
$$

We first notice the (easy and left to the reader) identity

$$
\left\langle v \nabla_{v} A(v)\right\rangle_{\mathbb{S}^{n-1}}=-\langle A(v)\rangle_{\mathbb{S}^{n-1}},
$$


which is valid for any smooth tangent vector field $A(v)$ on $\mathbb{S}^{n-1}$. Now, we form the vector test function

$$
\varphi_{f}(v)=\frac{P_{\Omega_{f}^{\perp}} v}{\left|P_{\Omega_{f}^{\perp}} v\right|}
$$

We note that $\varphi_{f}$ can be written:

$$
\varphi_{f}(v)=\Psi\left(v \cdot \Omega_{f}\right) P_{\Omega_{f}^{\perp}} v, \quad \Psi(X)=\frac{1}{\left(1-X^{2}\right)^{1 / 2}}, \quad X=v \cdot \Omega_{f} \in[-1,1] .
$$

The following identity holds:

$$
\left\langle\varphi_{f} \nabla_{v} \cdot\left(P_{v^{\perp}} \Omega_{f} f\right)\right\rangle_{\mathbb{S}^{n-1}}=0,
$$

for all distribution function $f(v)$ on $\mathbb{S}^{n-1}$. This identity is proved at Lemma 2.4 below.

Now, first we note that, because of proposition 2.1, we have

$$
f^{\varepsilon}(x, v, t) \rightarrow \rho(x, t) \delta_{\Omega(x, t)}(v) \quad \text { as } \quad \varepsilon \rightarrow 0 .
$$

In other words, in the limit $\varepsilon \rightarrow 0, f^{\varepsilon}$ converges to a monokinetic distribution function.

Now, we integrate (2.21) upon $v \in \mathbb{S}^{n-1}$. By Green's formula, the integrals of the last term of the left-hand side and of the right-hand side vanish and we are left with the mass conservation equation

$$
\left(\rho_{f^{\varepsilon}}\right)_{t}+\nabla_{x} \cdot\left(\rho_{f_{\varepsilon}} u_{f_{\varepsilon}}\right)=0 .
$$

Now, taking the limit $\varepsilon \rightarrow 0$ and using (2.33), we have $u_{f \varepsilon} \rightarrow u=\Omega$ and we finally get (2.28).

In order to find an equation for $\Omega$, we multiply (2.21) by the GCI (2.31) and get

$$
\left\langle\left(f_{t}^{\varepsilon}+v \cdot \nabla_{x} f^{\varepsilon}+\frac{1}{\rho_{f^{\varepsilon}}\left|u_{f^{\varepsilon}}\right|} \nabla_{v} \cdot\left[\left(P_{v^{\perp}} \ell_{f^{\varepsilon}}\right) f^{\varepsilon}\right]\right) \varphi_{f^{\varepsilon}}\right\rangle_{\mathbb{S}^{n-1}}=0 .
$$

Now, we intend to pass to the limit $\varepsilon \rightarrow 0$ in this equation. However, notice that the GCI (2.31) is singular near $v=\Omega$ while $f^{\varepsilon}$ concentrates to a Dirac delta at this point. Therefore, this limit is not straightforward and requires some smoothing procedure. Instead, in the next section, we consider a noisy version of this problem such that $f^{\varepsilon}$ converges to a smooth distribution, which allows to pass to the limit easily (see eq. (2.41)). If we let $\delta \rightarrow 0$ in eq. (2.41) below, we get the momentum balance equation (2.29). The direct proof from using (2.34) is left to future work.

LEMMA 2.4. The following identity holds:

$$
\left\langle\varphi_{f} \nabla_{v} \cdot\left(P_{v^{\perp}} \Omega_{f} f\right)\right\rangle_{\mathbb{S}^{n-1}}=0,
$$

for all distribution function $f(v)$ on $\mathbb{S}^{n-1}$.

Proof. We omit the subscript $f$ to $\Omega$, the dependence of $\Psi$ upon $(v \cdot \Omega)$ and the indices $\mathbb{S}^{n-1}$ to the brackets for the sake of simplicity. We denote by $B$ the quantity to be evaluated, i.e;

$$
B=\left\langle\Psi P_{\Omega^{\perp}} v \nabla_{v} \cdot\left(P_{v^{\perp}} \Omega f\right)\right\rangle .
$$


Pulling $P_{\Omega^{\perp}}$ outside the bracket and using Leibnitz formula, we have:

$$
\begin{aligned}
B=P_{\Omega^{\perp}}\langle\Psi v & \left.\nabla_{v} \cdot\left(P_{v^{\perp}} \Omega f\right)\right\rangle \\
& =P_{\Omega^{\perp}}\left\langle v \nabla_{v} \cdot\left(\Psi P_{v^{\perp}} \Omega f\right)\right\rangle-P_{\Omega^{\perp}}\left\langle v \nabla_{v} \Psi \cdot P_{v^{\perp}} \Omega f\right\rangle \\
& =-P_{\Omega^{\perp}}\left\langle\Psi P_{v^{\perp}} \Omega f\right\rangle-P_{\Omega^{\perp}}\left\langle v \Psi^{\prime}\left|P_{v^{\perp}} \Omega\right|^{2} f\right\rangle,
\end{aligned}
$$

where we have used (2.30) and the fact that $\nabla_{v} \Psi=\Psi^{\prime} P_{v^{\perp}} \Omega$ to simplify the first and second brackets in the last equation. We denote by $\Psi^{\prime}(X)$ the derivative or $\Psi(X)$ with respect to $X$. Now, we remark that

$$
\begin{aligned}
P_{\Omega^{\perp}} P_{v^{\perp}} \Omega=(\operatorname{Id}-\Omega \otimes \Omega) & (\operatorname{Id}-v \otimes v) \Omega \\
& =-(v \cdot \Omega)(\operatorname{Id}-\Omega \otimes \Omega) v=-(v \cdot \Omega) P_{\Omega^{\perp}} v,
\end{aligned}
$$

and that $\left|P_{v^{\perp}} \Omega\right|^{2}=1-(v \cdot \Omega)^{2}$. This leads to

$$
B=\left\langle P_{\Omega} \perp v\left((v \cdot \Omega) \Psi-\left(1-(v \cdot \Omega)^{2}\right) \Psi^{\prime}\right) f\right\rangle .
$$

Now, since $\Psi$ satisfies the differential equation

$$
X \Psi(X)-\left(1-X^{2}\right) \Psi^{\prime}(x)=0,
$$

we have $B=0$, which proves $(2.35)$.

REMARK 2.2. The $n-1$ independent functions $\left(\varphi_{f}\right)_{k}=\varphi_{f} \cdot e_{k}$ where $\left(e_{1}, \ldots, e_{n-1}\right)$ is an orthonormal basis of $\left\{\operatorname{Span}\left(\Omega_{f}\right)\right\}^{\perp}$ are the 'Generalized Collision Invariants' (or GCI) associated to the operator $Q(f)=-\nabla_{v} \cdot\left(P_{v^{\perp}} \Omega_{f} f\right)$ in the sense of [13] (see proof of Theorem 2.5 below for more detail on the GCI).

REMARK 2.3. The quantity $\mathcal{F}(f)$ is a free energy for the spatially homogeneous problem (2.22) and provides a variational structure. First, let us denote by $\nu=\frac{\delta \mathcal{F}}{\delta f}$ the first variation of $\mathcal{F}$ with respect to $f$. It is defined by

$$
\left\langle\frac{\delta \mathcal{F}}{\delta f}, g\right\rangle=\left.\frac{d}{d \epsilon}\right|_{\epsilon=0} \mathcal{F}(f+\epsilon g),
$$

where $g$ is an increment of $f$, i.e. a function $g(v)$ satisfying $\int g(v) d v=0$ (so that $f+\epsilon g$ satisfies the admissibility condition $\left.\int(f+\epsilon g) d v=1\right)$ and such that there exists $\epsilon_{0}$ with $f+\epsilon g \geq 0$ for all $\epsilon<\epsilon_{0}$. Eq. (2.22) can be recast as

$$
f_{t}-\frac{1}{\varepsilon|u|} \nabla_{v} \cdot\left\{\left[\nabla_{v}\left(\frac{\delta \mathcal{F}}{\delta f}\right)\right] f\right\}=0,
$$

which shows that the flow of (2.22) has a gradient flow structure in the Wasserstein metric [42]. We have:

$$
\mathcal{F}(f)_{t}+\frac{1}{\varepsilon|u|} \int\left|\nabla_{v}\left(\frac{\delta \mathcal{F}}{\delta f}\right)\right|^{2} f d v=0,
$$

which provides another proof of the decay of $\mathcal{F}(f)$ with time. Indeed, we have:

$$
\frac{\delta \mathcal{F}}{\delta f}(g)=-2 u \cdot \int g(v) v d v=\langle-2(u \cdot v), g\rangle
$$


which yields

$$
\nu=\frac{\delta \mathcal{F}}{\delta f}=-2 u \cdot v .
$$

A simple computation shows that

$$
\nabla_{v} \nu=-P_{v^{\perp}} u=-|u| P_{v^{\perp}} \Omega .
$$

Therefore, eq. (2.22) can be written as (2.36). Now, multiplying by $\frac{\delta \mathcal{F}}{\delta f}$, integrating over $v$ and using Green's formula, we get (2.37). The variational structure of (2.22) will be exploited in future work.

2.4. Local interaction scaling with noise. In this scaling we assume that $\delta \neq 0$ is a given constant. We also assume that $\varepsilon$ and $\eta$ are such that:

$$
\varepsilon \rightarrow 0, \quad \eta \rightarrow 0, \quad \frac{\eta^{2}}{\varepsilon} \rightarrow 0 .
$$

With this last assumption, the $O\left(\frac{\eta^{2}}{\varepsilon}\right)$ term in (2.20), which results from the nonlocality of the average alignment direction, vanishes. Therefore, this scaling keeps only the local contribution of the alignment interaction. The resulting asymptotic problem, keeping only terms of order $\mathcal{O}(1)$ or larger, is written:

$$
f_{t}^{\varepsilon}+v \cdot \nabla_{x} f^{\varepsilon}=\frac{1}{\varepsilon}\left\{-\nabla_{v} \cdot\left[\left(P_{v^{\perp}} \Omega_{\varepsilon}\right) f^{\varepsilon}\right]+\delta \Delta_{v} f^{\varepsilon}\right\} .
$$

The difference with (2.20) it that the second term at the right-hand side of $(2.20)$ is omitted. The limit of (2.38) as $\varepsilon \rightarrow 0$ has been studied in [13] in dimension 3 and in [19] in any dimensions. The result is stated in the following theorem.

ThEOREM 2.5. If $\varepsilon$ tends to 0 in (2.38), we formally have $f^{\varepsilon} \rightarrow \rho M_{\Omega}$ where $M_{\Omega}(v)$ is the Von Mises-Fischer distribution:

$$
M_{\Omega}(v)=\frac{\exp (\beta(v \cdot \Omega)) d v}{\int_{v \in \mathbb{S}^{n-1}} \exp (\beta(v \cdot \Omega)) d v}, \quad \beta=\frac{1}{\delta},
$$

and $\rho$ and $\Omega$ satisfy the following Self-Organized Hydrodynamic (SOH) system:

$$
\begin{aligned}
& \partial_{t} \rho+c_{1} \nabla_{x} \cdot(\rho \Omega)=0, \\
& \rho\left(\partial_{t} \Omega+c_{2} \Omega \cdot \nabla_{x} \Omega\right)+\delta P_{\Omega^{\perp}} \nabla_{x} \rho=0 .
\end{aligned}
$$

The constants $c_{1}$ and $c_{2}$ are defined by

$$
\begin{aligned}
& c_{1}=\int_{v \in \mathbb{S}^{n-1}} M_{\Omega}(v)(v \cdot \Omega) d v \\
& c_{2}=\frac{\int_{v \in \mathbb{S}^{n-1}} M_{\Omega}(v) h(v \cdot \Omega)\left(1-(v \cdot \Omega)^{2}\right)(v \cdot \Omega) d v}{\int_{v \in \mathbb{S}^{n-1}} M_{\Omega}(v) h(v \cdot \Omega)\left(1-(v \cdot \Omega)^{2}\right) d v},
\end{aligned}
$$

where $h(v \cdot \Omega)$ is the Generalized Collision Invariants (GCI) [13] and is defined as follows in the $n$-dimensional case [19]. Set $\psi_{\xi}(v)=h(\Omega \cdot v)(\xi \cdot v)$ where $\xi \in \mathbb{R}^{n}$ is any vector such that $\xi \cdot \Omega=0$. Then, $\psi_{\xi}$ is the unique solution in the Sobolev space $H^{1}\left(\mathbb{S}^{n-1}\right)$ with zero mean, of the following elliptic problem:

$$
-\Delta_{v} \psi-\beta\left(\Omega \cdot \nabla_{v}\right) \psi=\xi \cdot v .
$$


Finally, we formally have $|\Omega(\cdot, t)|=1$ for all $t>0$ as soon as the initial data $\left(\rho_{0}, \Omega_{0}\right)$ satisfies $\left|\Omega_{0}\right|=1$.

Sketch of the proof. We refer to [13] in the three dimensional case and [19] in the general $n$-dimensional case for more details. We denote by

$$
Q(f)=-\nabla_{v} \cdot\left[\left(P_{v^{\perp}} \Omega_{f}\right) f\right]+\delta \Delta_{v} f, \quad \Omega_{f}=\frac{j_{f}}{\left|j_{f}\right|}, \quad j_{f}=\int f(v) v d v
$$

the 'collision' operator and write (2.38) as

$$
f_{t}^{\varepsilon}+v \cdot \nabla_{x} f^{\varepsilon}=\frac{1}{\varepsilon} Q\left(f^{\varepsilon}\right) .
$$

We assume that $f^{\varepsilon} \rightarrow f$ as $\varepsilon \rightarrow 0$ as smoothly as needed (which means in particular that derivatives of $f^{\varepsilon}$ converge to the corresponding derivatives of $f$ ). From (2.45), we notice that $Q\left(f^{\varepsilon}\right)=\mathcal{O}(\varepsilon)$, which implies that $Q(f)=0$. The functions $\varphi(v)$ such that $Q(\varphi)=0$ are the so-called equilibria of $Q$. It is shown in $[13,19]$ that the equilibria of $Q$ are of the form $\varphi(v)=\rho M_{\Omega}(v)$ with $M_{\Omega}$ given by (2.39) and $\rho>0, \Omega \in \mathbb{S}^{n-1}$ are arbitrary. Therefore,

$$
f(x, v, t)=\rho(x, t) M_{\Omega(x, t)}(v),
$$

where $\rho(x, t)$ and $\Omega(x, t)$ need now to be determined.

In order to find equations for $\rho$ and $\Omega$, we introduce the notion of a 'Generalized Collision Invariant' or GCI. In kinetic theory, a 'Collision Invariant' (or CI) of the collision operator $Q$ is a function $\psi(v)$ such that

$$
\int Q(f)(v) \psi(v) d v=0, \quad \forall \text { functions } f(v) .
$$

In this formal proof, we do not specify the functional setting any further. Here, following this definition, we find that the set of CI's is the one-dimensional space spanned by the constants. But the dimension of the space of the CI's determines the number of conservation equations for the macroscopic model. Here, we only get one conservation equation (namely the mass conservation equation, which determines $\rho$ ) with this concept. We are lacking $n-1$ independent equations to determine the unit vector $\Omega$. In order to find these additional equations, we weaken the concept of a CI to define the concept of a GCI. For this purpose, we introduce the operator

$$
\mathcal{Q}(\Omega, f)=-\nabla_{v} \cdot\left[\left(P_{v^{\perp}} \Omega\right) f\right]+\delta \Delta_{v} f .
$$

We note that for fixed $\Omega, \mathcal{Q}(\Omega, f)$ is a linear operator of $f$. The GCI's are now defined as follows:

DEFINITION 2.6. Let $\Omega \in \mathbb{S}^{n-1}$ be given. The function $\psi_{\Omega}(v)$ is a 'Generalized Collision Invariant' or GCI of $Q$ associated to the direction $\Omega$ if and only if we have

$$
\int \mathcal{Q}(\Omega, f) \psi_{\Omega}(v) d v=0, \quad \forall \text { functions } f(v) \text { such that } \Omega_{f}= \pm \Omega .
$$

The constraint $\Omega_{f}= \pm \Omega$ is a linear constraint on $f$ and it is shown in $[13,19]$ that condition (2.47) is equivalent to saying that there exists a vector $\xi \in \mathbb{R}^{n}$, with $\xi \cdot \Omega=0$ such that $\psi_{\Omega}=\psi_{\xi}$ solves the equation:

$$
\mathcal{Q}^{*}\left(\Omega, \psi_{\xi}\right)=\xi \cdot v,
$$


where $\mathcal{Q}^{*}(\Omega, \cdot)$ is the $L^{2}$-adjoint of $\mathcal{Q}(\Omega, \cdot)$. We easily see that $(2.48)$ leads to $(2.44)$. Furthermore, by rotational invariance, $\psi_{\xi}$ is, up to a constant, of the form $h(\Omega \cdot v)(\xi \cdot v)$. Therefore, the vector space of GCI's is spanned by the function 1 and the $n-1$ independent functions $h(\Omega \cdot v) v \cdot e_{k}$, where $\left(e_{1}, \ldots, e_{k}, \ldots, e_{n-1}\right)$ is an orthonormal basis of $(\operatorname{Span}\{\Omega\})^{\perp}$.

Now, (2.40) and (2.41) are obtained by pre-multiplying (2.38) by respectively 1 (for (2.40)) and a non-constant GCI $\psi_{\Omega_{f_{\varepsilon}}}$ (for (2.41)) and integrating them with respect to $v$. By (2.47), the contributions of the singular term involving $Q$ disappear and we can let $\varepsilon \rightarrow 0$ in the resulting equations, which only involve regular terms in $\varepsilon$. After some analytic computations which are detailed in $[13,19]$, we find eqs. (2.40), (2.41) together with the coefficients $(2.42)$, (2.43).

REMARK 2.4. We note that $c_{1}$ is such that $0<c_{1}<1$. The parameter $c_{1}$ is the classical order parameter of the Von-Mises distribution. The parameter $c_{2}$ satisfies $0<c_{2}<c_{1}$. In more general situations (involving a limited field of vision), the range of values of $c_{2}$ may be much larger. It can be negative or larger than $c_{1}$ (see [19] for details).

2.5. Weakly non-local interaction scaling with noise. In this section, we propose a scaling which unifies the two previous ones. In this scaling we assume that $\delta$ is a given constant and that:

$$
\varepsilon \rightarrow 0, \quad \eta \rightarrow 0, \quad \frac{\eta^{2}}{\varepsilon} \rightarrow 1
$$

Here $\frac{\eta^{2}}{\varepsilon} \rightarrow 1$ instead of 0 like in the previous section. Inserting these assumptions into $(2.20)$, and keeping terms of order $\mathcal{O}(1)$ or larger, we get

$$
\begin{aligned}
f_{t}^{\varepsilon}+v \cdot \nabla_{x} f^{\varepsilon}+\frac{1}{\rho_{f^{\varepsilon}}\left|u_{f^{\varepsilon}}\right|} \nabla_{v} \cdot\left[\left(P_{v^{\perp}} \ell_{f^{\varepsilon}}\right) f^{\varepsilon}\right]= \\
\\
\frac{1}{\varepsilon}\left\{-\nabla_{v} \cdot\left[\left(P_{v^{\perp}} \Omega_{f^{\varepsilon}}\right) f^{\varepsilon}\right]+\delta \Delta_{v} f^{\varepsilon}\right\} .
\end{aligned}
$$

The difference with (2.20) it that the second term at the right-hand side of $(2.20)$ is moved to the left-hand side while making $\eta^{2} / \varepsilon=1$. The limit of (2.49) as $\varepsilon \rightarrow 0$ can been studied by the same techniques as in $[13,19]$ (see also proof of Theorem 2.5). The result is stated in the following theorem.

THEOREM 2.7. If $\varepsilon$ tends to 0 in (2.49), we formally have $f^{\varepsilon} \rightarrow \rho M_{\Omega}$ where $M_{\Omega}(v)$ is the Von Mises-Fischer distribution (2.39). $\rho$ and $\Omega$ satisfy the following viscous $\mathrm{SOH}$ system:

$$
\begin{aligned}
& \partial_{t} \rho+c_{1} \nabla_{x} \cdot(\rho \Omega)=0, \\
& \rho\left(\partial_{t} \Omega+c_{2} \Omega \cdot \nabla_{x} \Omega\right)+\left(\delta+c_{3} \Theta\right) P_{\Omega^{\perp}} \nabla_{x} \rho=c_{3} k c_{1} P_{\Omega^{\perp}} \Delta_{x}(\rho \Omega),
\end{aligned}
$$

where the constants $c_{1}$ and $c_{2}$ are defined as in Theorem 2.5 and

$$
c_{3}=\frac{(n-1) \delta+c_{2}}{c_{1}}
$$

We formally have $|\Omega(\cdot, t)|=1$ for all $t>0$ as soon as the initial data $\left(\rho_{0}, \Omega_{0}\right)$ satisfies $\left|\Omega_{0}\right|=1$. 
REMARK 2.5. We notice that $c_{k} \rightarrow 1$ as $\delta \rightarrow 0$ for $k=1,2,3$ and we recover the noiseless system (2.28), (2.29) when $\delta \rightarrow 0$.

Proof. We write (2.49) as

$$
\left(T_{1}+T_{2}\right) f^{\varepsilon}=\frac{1}{\varepsilon} Q\left(f^{\varepsilon}\right),
$$

where $T_{1}+T_{2}$ and $Q$ are respectively the operators appearing at the left and right hand sides of (2.49). $T_{1}=\partial_{t}+v \cdot \nabla_{x}$ and $T_{2}$ is the remaining part of the left-hand side. Integrating over $v$ and letting $f^{\varepsilon} \rightarrow \rho M_{\Omega}$ leads to the mass conservation equation (2.40) unchanged, since $T_{2}$ is in divergence form and vanishes through integration with respect to $v$.

Now, to get the momentum equation, we proceed like in $[13,19]$ or as in the proof of Theorem 2.5. From the Generalized Collision Invariant property, it follows that

$$
P_{\Omega^{\perp}} \int_{v \in \mathbb{S}^{n-1}} T\left(\rho M_{\Omega}\right) h v d v=0 .
$$

Now, the term

$$
P_{1}:=P_{\Omega^{\perp}} \int_{v \in \mathbb{S}^{n-1}} T_{1}\left(\rho M_{\Omega}\right) h v d v
$$

gives rise to the same expression as in Theorem 2.5. This expression is

$$
P_{1}=\beta \alpha \rho \partial_{t} \Omega+\gamma \Omega \cdot \nabla_{x} \Omega+\alpha P_{\Omega^{\perp}} \nabla_{x} \rho,
$$

with

$$
\begin{aligned}
& \alpha=\frac{1}{n-1} \int_{v \in \mathbb{S}^{n-1}} M_{\Omega}(v) h\left(1-(v \cdot \Omega)^{2}\right) d v \\
& \gamma=\frac{1}{(n-1) \delta} \int_{v \in \mathbb{S}^{n-1}} M_{\Omega}(v) h\left(1-(v \cdot \Omega)^{2}\right)(v \cdot \Omega) d v .
\end{aligned}
$$

Dividing by $\alpha \beta$, we find the coefficients $c_{2}$ and $\delta$ of $(2.41)$ (we recall that $\beta \delta=1$ ).

We introduce the notation

$$
\ell:=\ell_{\rho M_{\Omega}}=P_{\Omega^{\perp}}\left(k c_{1} \Delta_{x}(\rho \Omega)-\Theta \nabla_{x} \rho\right),
$$

and consider

$$
\begin{aligned}
P_{2} & :=P_{\Omega^{\perp}} \int_{v \in \mathbb{S}^{n-1}} T_{2}\left(\rho M_{\Omega}\right) h v d v \\
& =\frac{1}{c_{1}} P_{\Omega^{\perp}} \int_{v \in \mathbb{S}^{n-1}} \nabla_{v} \cdot\left[\left(P_{v^{\perp}} \ell\right) M_{\Omega}\right] h v d v .
\end{aligned}
$$

Using Green's formula, we get

$$
P_{2}=-\frac{1}{c_{1}} P_{\Omega^{\perp}} \int_{v \in \mathbb{S}^{n-1}}\left[P_{v^{\perp}} \ell\right] \cdot \nabla_{v}(h v) M_{\Omega} d v .
$$

We note that $\left(P_{v^{\perp}} \ell\right) \cdot \nabla_{v} \varphi=\ell \cdot \nabla_{v} \varphi$, with $\varphi$ being any component of $h v$. We deduce that

$$
\begin{aligned}
P_{2} & =-\frac{1}{c_{1}} P_{\Omega^{\perp}} \int_{v \in \mathbb{S}^{n-1}}\left(\ell \cdot \nabla_{v}\right)(h v) M_{\Omega} d v \\
& =-\frac{1}{c_{1}} P_{\Omega^{\perp}}\left(\int_{v \in \mathbb{S}^{n-1}} \nabla_{v}(h v) M_{\Omega} d v\right)^{T} \ell .
\end{aligned}
$$


Now, we use the formulas:

$$
\begin{aligned}
& \int_{\mathbb{S}^{n-1}} \nabla_{v} g d v=(n-1) \int_{\mathbb{S}^{2}} v g d v \\
& \int_{\mathbb{S}^{n-1}}\left(\nabla_{v} g\right) h d v=(n-1) \int_{\mathbb{S}^{2}} v g h d v-\int_{\mathbb{S}^{2}}\left(\nabla_{v} h\right) g d v
\end{aligned}
$$

for any pair of scalar functions $g, h$ on $\mathbb{S}^{n-1}$. We recall that $\nabla_{v} M_{\Omega}=\beta P_{v} \Omega M_{\Omega}$. Since $\ell \cdot \Omega=0$, we compute the matrix

$$
\begin{aligned}
D & :=\left(\int_{v \in \mathbb{S}^{n-1}} \nabla_{v}(h v) M_{\Omega} d v\right)^{T} P_{\Omega^{\perp}} \\
& =(n-1)\left(\int_{v \in \mathbb{S}^{n-1}}(v \otimes v) h M_{\Omega} d v\right) P_{\Omega^{\perp}} \\
& \quad-\beta\left(\int_{v \in \mathbb{S}^{n-1}}\left(v \otimes P_{v^{\perp}} \Omega\right) h M_{\Omega} d v\right) P_{\Omega^{\perp}} \\
& :=(n-1) D_{1}-D_{2} .
\end{aligned}
$$

We decompose

$$
v=v_{\perp}+v_{\|}, \quad v_{\perp}=P_{\Omega^{\perp}} v, \quad v_{\|}=(v \cdot \Omega) \Omega .
$$

Using this decomposition and the fact that integrals of odd degree polynomials of $v_{\perp}$ over $\mathbb{S}^{n-1}$ vanish, we have:

$$
D_{1}=\left(\int_{v \in \mathbb{S}^{n-1}}\left(v_{\perp} \otimes v_{\perp}\right) h M_{\Omega} d v\right) P_{\Omega^{\perp}}=\alpha P_{\Omega^{\perp}},
$$

and

$$
D_{2}=\beta\left(\int_{v \in \mathbb{S}^{n-1}}\left(v_{\|}+v_{\perp}\right) \otimes\left(\Omega-(\Omega \cdot v)\left(v_{\|}+v_{\perp}\right)\right) h M_{\Omega} d v\right) P_{\Omega^{\perp}} .
$$

Owing to the fact that any term of the form $\left(A \otimes v_{\|}\right) P_{\Omega^{\perp}}=0$ for any vector $A$, we have since $v_{\|}$is parallel to $\Omega$ :

$$
D_{2}=-\beta\left(\int_{v \in \mathbb{S}^{n-1}}\left(v_{\perp} \otimes v_{\perp}\right)(\Omega \cdot v) h M_{\Omega} d v\right) P_{\Omega^{\perp}}=-\gamma P_{\Omega^{\perp}} .
$$

Inserting these results into (2.53), we get

$$
P_{2}=-\frac{(n-1) \alpha+\gamma}{c_{1}} \ell
$$

Collecting all the results and dividing by $\alpha \beta$, we are led to the momentum equation (2.51), which ends the proof.

2.6. Strong potential force scaling: induced capillary (or Korteweg) force. In this section, we investigate the case where the attraction-repulsion force term is of the same order as the alignment term in the expression of the alignment direction $v_{f}^{\eta}$, i.e. we change $v_{f}^{\eta}$ into $\tilde{v}_{f}^{\eta}$ in (2.15), with $\tilde{v}_{f}^{\eta}$ given by:

$$
\tilde{v}_{f}^{\eta}=\frac{j_{f}^{\eta}+r_{f}^{\eta}}{\left|j_{f}^{\eta}+r_{f}^{\eta}\right|}
$$


where $j_{f}^{\eta}$ and $r_{f}^{\eta}$ are respectively given by (2.17) and (2.18). Note that, by contrast to (2.16), there is no $\eta^{2}$ in front of $r_{f}^{\eta}$ in (2.54).

The Taylor expansion of $\tilde{v}_{f}^{\eta}$ is now given by

$$
\begin{aligned}
& \tilde{v}_{f}^{\eta}=\hat{\Omega}_{f}+\eta^{2} \frac{1}{\rho_{f}\left|u_{f}\right|} \ell_{f}+o\left(\eta^{2}\right), \\
& \hat{\Omega}_{f}=\frac{u_{f}-\Theta \nabla_{x} \rho_{f}}{\left|u_{f}-\Theta \nabla_{x} \rho_{f}\right|}, \quad \ell_{f}=P_{\hat{\Omega}_{f}^{\perp}}\left(k \Delta_{x}\left(\rho_{f} u_{f}\right)-\Theta_{2} \nabla_{x} \Delta_{x} \rho_{f}\right),
\end{aligned}
$$

where

$$
\frac{1}{2 n} \int_{x^{\prime} \in \mathbb{R}^{n}} \Phi(|\xi|)|\xi|^{2} d \xi=\Theta_{2}
$$

Here, we suppose like in [2], that the potential is repulsive at short scales and attractive at large scales (see Fig. 3). Therefore, $\Phi(|\xi|)$ is supposed to decrease for $|\xi| \in\left[0, \xi_{*}\right]$ and to increase for $|\xi| \in\left[\xi_{*},+\infty\right)$. Furthermore, since $\Phi(|\xi|)$ is supposed integrable on $\mathbb{R}^{n}$, we have $\Phi(|\xi|) \rightarrow 0$ as $|\xi| \rightarrow \infty$. It results that $\Phi\left(\xi_{*}\right)<0$ and that $\Phi \geq 0$ for $|\xi| \in\left[0, \xi_{0}\right]$ and $\Phi \leq 0$ for $|\xi| \in\left[\xi_{0},+\infty\right)$ where $\xi_{0}<\xi_{*}$. We make the additional assumption that the zero-th order moment vanishes:

$$
\Theta=0,
$$

which expresses the balance between the attractive and repulsive parts of $\Phi$. Given the above assumptions, the second moment is negative:

$$
\Theta_{2}<0 \text {. }
$$

Equivalently, we can modify the expression of the attraction-repulsion (2.18) as:

$$
r_{f}^{\eta}=-\nabla_{x} \int_{\left(x^{\prime}, v^{\prime}\right) \in \mathbb{R}^{n} \times \mathbb{S}^{n-1}} \Phi\left(\frac{\left|x^{\prime}-x\right|}{\eta}\right)\left(f\left(x^{\prime}, v^{\prime}, t\right)-f(x, v, t)\right) d x^{\prime} d v^{\prime},
$$

instead of making the assumption (2.55). This modification of the interaction potential was first introduced by van der Waals [39]. In this case, the inequality (2.56) has to be put as an assumption.

With these assumptions, the Taylor expansion of $v_{f}^{\eta}$ simplifies and becomes:

$$
\begin{aligned}
& \tilde{v}_{f}^{\eta}=\Omega_{f}+\eta^{2} \frac{1}{\rho_{f}\left|u_{f}\right|} \ell_{f}+o\left(\eta^{2}\right), \\
& \Omega_{f}=\frac{u_{f}}{\left|u_{f}\right|}, \quad \ell_{f}=P_{\Omega_{f}^{\perp}}\left(k \Delta_{x}\left(\rho_{f} u_{f}\right)-\Theta_{2} \nabla_{x} \Delta_{x} \rho_{f}\right) .
\end{aligned}
$$

Now, we can develop the same theory as before, assuming that

$$
\varepsilon \rightarrow 0, \quad \eta \rightarrow 0, \quad \frac{\eta^{2}}{\varepsilon} \rightarrow 1 .
$$

Inserting these assumptions into (2.20), and keeping terms of order $\mathcal{O}(1)$ or larger, we get

$$
\begin{aligned}
f_{t}^{\varepsilon}+v \cdot \nabla_{x} f^{\varepsilon}+\frac{1}{\rho_{f^{\varepsilon}}\left|u_{f^{\varepsilon}}\right|} \nabla_{v} \cdot\left[\left(P_{v^{\perp}} \ell_{f^{\varepsilon}}\right) f^{\varepsilon}\right]= \\
\\
\frac{1}{\varepsilon}\left\{-\nabla_{v} \cdot\left[\left(P_{v^{\perp}} \Omega_{f^{\varepsilon}}\right) f^{\varepsilon}\right]+\delta \Delta_{v} f^{\varepsilon}\right\} .
\end{aligned}
$$




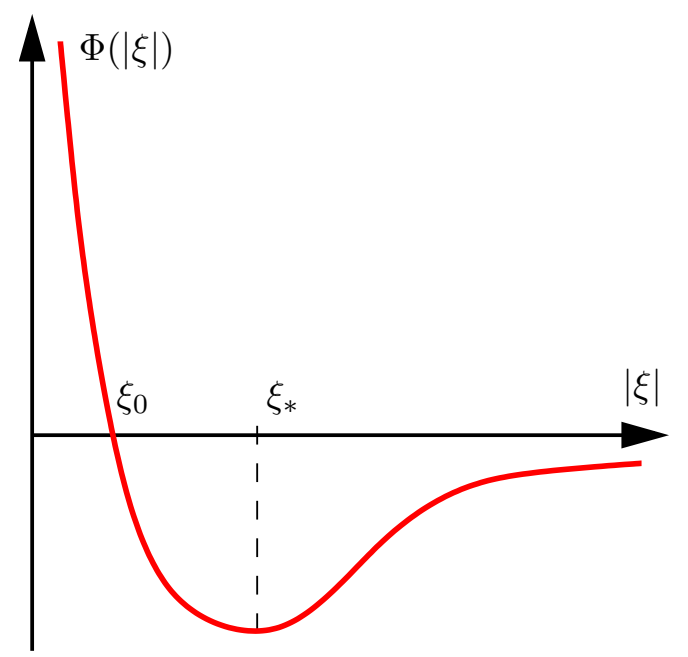

FIG. 3. The attraction-repulsion potential $\Phi$.

with $\ell_{f}$ given by (2.58). The limit $\varepsilon \rightarrow 0$ can be performed like in Section 2.5 and we obtain the following theorem:

THEOREM 2.8. If $\varepsilon$ tends to 0 in (2.59) and under the assumption (2.55) (if the expression (2.18) of the potential force is used) or (2.56) (if the expression (2.57) is used), we formally have $f^{\varepsilon} \rightarrow \rho M_{\Omega}$ where $M_{\Omega}(v)$ is the Von Mises-Fischer distribution (2.39). $\rho$ and $\Omega$ satisfy the viscous SOH system with capillary (or Korteweg) force term:

$$
\begin{aligned}
& \partial_{t} \rho+c_{1} \nabla_{x} \cdot(\rho \Omega)=0 \\
& \rho\left(\partial_{t} \Omega+c_{2} \Omega \cdot \nabla_{x} \Omega\right)+\delta P_{\Omega^{\perp}} \nabla_{x} \rho=c_{3} k c_{1} P_{\Omega^{\perp}} \Delta_{x}(\rho \Omega)+c_{3}\left|\Theta_{2}\right| P_{\Omega^{\perp}} \nabla_{x} \Delta_{x} \rho,
\end{aligned}
$$

where the constants $c_{1}$ and $c_{2}$ are defined as in Theorem 2.5 and $c_{3}$ as in Theorem 2.7. We formally have $|\Omega(\cdot, t)|=1$ for all $t>0$ as soon as the initial data $\left(\rho_{0}, \Omega_{0}\right)$ satisfies $\left|\Omega_{0}\right|=1$.

REMARK 2.6. The last term at the right-hand side of (2.61) has the same expression as the capillary force (or Korteweg term [23]) in fluid dynamics, except for the projection operator $P_{\Omega^{\perp}}$. This capillary force is induced by the attractive part of the potential $\Phi$.

\section{Existence theory.}

3.1. Existence in $2 \mathrm{D}$ with viscosity. This section is concerned with a local existence result in $2 \mathrm{D}$ for an $\mathrm{SOH}$ system of the general form

$$
\begin{aligned}
& \partial_{t} \rho+\nabla_{x} \cdot(\rho \Omega)=0, \\
& \rho\left(\partial_{t} \Omega+c \Omega \cdot \nabla_{x} \Omega\right)+P_{\Omega^{\perp}} \nabla_{x}(p(\rho))=\mu P_{\Omega^{\perp}} \Delta_{x}(\rho \Omega),
\end{aligned}
$$

where the constants $c \in \mathbb{R}$ and $\mu \geq 0$ are given and the pressure relation $p(\rho)$ satisfies $p^{\prime}(\rho)>0$. All systems derived in the previous section can by recast in this form, with a particular choice of $c, \mu$ and $p(\rho)$, after time rescaling, except for the last one (Section 
2.6) involving the capillary force. The system is supplemented with initial data $\rho_{0}>0$ and $\Omega_{0}$ such that $\left|\Omega_{0}\right|=1$. Because of the presence of the projection operator $P_{\Omega^{\perp}}$, this unit norm constraint $|\Omega(x, t)|=1$ is preserved by the dynamics but at the price of a loss of conservativity. Because of this, a notion of entropy condition for shock solutions is open. In [29], a selection criterion based on a numerical comparison with the particle system is proposed. It is based on the approximation of this system by a conservative system with a stiff relaxation right-hand side. This relaxation approach is also quite common in the Ericksen-Leslie theory of liquid crystals [25, 26] and has been recently extended to convex constraints [16].

We assume that the domain is the square box $\Pi^{2}=[0,1]^{2}$ with periodic boundary conditions. The consideration of physical boundary conditions for the short-time existence of smooth solutions of quasi-linear symmetrizable hyperbolic systems is rather technical. The strictly dissipative property is required for the boundary condition [34]. Here the assumption of periodic boundary condition allows to avoid these complications which are not the purpose of this paper.

THEOREM 3.1. We assume that the initial data belong to $H^{m}\left(\Pi^{2}\right)$ with $m>2$. Then, there exists a time $T>0$ and a unique solution $(\rho, \varphi)$ of the system (3.1)-(3.2) in $L^{\infty}\left([0, T], H^{m}\left(\Pi^{2}\right)\right) \cap H^{1}\left([0, T], H^{m-1}\left(\Pi^{2}\right)\right)$ such that $\rho$ remains positive. If, in addition, $\mu>0$, then, the solution also belongs to $L^{2}\left([0, T], H^{m+1}\left(\Pi^{2}\right)\right)$.

Proof. In $2 \mathrm{D}$, we can set $\Omega=(\cos \varphi, \sin \varphi)$. We recall that

$$
\partial_{t} \Omega=\Omega^{\perp} \partial_{t} \varphi, \quad \nabla_{x} \cdot \Omega=\left(\Omega^{\perp} \cdot \nabla_{x}\right) \varphi, \quad P_{\Omega^{\perp}}=\Omega^{\perp} \otimes \Omega^{\perp},
$$

with $\Omega^{\perp}=(-\sin \varphi, \cos \varphi)$. Then, we have

$$
\begin{aligned}
& \Delta_{x}(\rho \Omega)=\Delta_{x} \rho \Omega+2 \Omega^{\perp}\left(\nabla_{x} \rho \cdot \nabla_{x} \varphi\right)-2 \rho \Omega\left|\nabla_{x} \varphi\right|^{2}+\rho \Omega^{\perp} \Delta_{x} \varphi \\
& \Omega^{\perp} \cdot \Delta_{x}(\rho \Omega)=\rho \Delta_{x} \varphi+2\left(\nabla_{x} \rho \cdot \nabla_{x} \varphi\right) .
\end{aligned}
$$

Therefore, system (3.1), (3.2) is written:

$$
\begin{aligned}
& \left(\partial_{t}+\Omega \cdot \nabla_{x}\right) \rho+\rho\left(\Omega^{\perp} \cdot \nabla_{x}\right) \varphi=0 \\
& \left(\partial_{t}+c \Omega \cdot \nabla_{x}\right) \varphi+\frac{p^{\prime}(\rho)}{\rho}\left(\Omega^{\perp} \cdot \nabla_{x}\right) \rho=\mu\left(\Delta_{x} \varphi+2 \frac{\nabla_{x} \rho \cdot \nabla_{x} \varphi}{\rho}\right) .
\end{aligned}
$$

Introduce $\hat{\rho}=a(\rho)$ and $\lambda(\hat{\rho})$ such that

$$
a^{\prime}(\rho)=\frac{\sqrt{p^{\prime}(\rho)}}{\rho}, \quad \lambda(\hat{\rho})=a^{\prime}(\rho) \rho, \quad h(\hat{\rho})=2 \ln \rho .
$$

Then, system (3.3), (3.4) becomes:

$$
\begin{aligned}
& \left(\partial_{t}+\Omega \cdot \nabla_{x}\right) \hat{\rho}+\lambda(\hat{\rho})\left(\Omega^{\perp} \cdot \nabla_{x}\right) \varphi=0, \\
& \left(\partial_{t}+c \Omega \cdot \nabla_{x}\right) \varphi+\lambda(\hat{\rho})\left(\Omega^{\perp} \cdot \nabla_{x}\right) \hat{\rho}=\mu\left(\Delta_{x} \varphi+\nabla_{x} h(\hat{\rho}) \cdot \nabla_{x} \varphi\right) .
\end{aligned}
$$

From (3.3), we have the following a priori estimate (maximum principle):

$$
\rho_{\min } \exp \left(-\int_{0}^{t}\left\|\nabla_{x} \varphi(\cdot, s)\right\|_{L^{\infty}\left(\Pi^{2}\right)} d s\right) \leq \rho \leq \rho_{\max } \exp \left(\int_{0}^{t}\left\|\nabla_{x} \varphi(\cdot, s)\right\|_{L^{\infty}\left(\Pi^{2}\right)} d s\right)
$$


where

$$
\rho_{\min }=\min _{x \in \Pi^{2}} \rho_{0}(x), \quad \rho_{\max }=\max _{x \in \Pi^{2}} \rho_{0}(x) .
$$

We remind the following lemma [41]:

Lemma 3.2. For any pair of functions $f, g$ in $H^{m}\left(\mathbb{R}^{n}\right) \cap L^{\infty}\left(\mathbb{R}^{n}\right)$, we have:

$$
\|f g\|_{H^{m}} \leq C\left(\|f\|_{H^{m}}\|g\|_{L^{\infty}}+\|f\|_{L^{\infty}}\|g\|_{H^{m}}\right) .
$$

If additionally, we suppose that $\nabla_{x} f \in L^{\infty}\left(\mathbb{R}^{n}\right)$, we have, for any $\alpha \in \mathbb{N}^{n}$, with $|\alpha|=\sum_{i=1}^{n} \alpha_{i}=m$ :

$$
\left\|D^{\alpha}(f g)-f D^{\alpha} g\right\|_{H^{m}} \leq C\left(\|f\|_{H^{m}}\|g\|_{L^{\infty}}+\left\|\nabla_{x} f\right\|_{L^{\infty}}\|g\|_{H^{m-1}}\right),
$$

where $D^{\alpha}=\partial_{x_{1}^{\alpha_{1}} \ldots x_{n}^{\alpha_{n}}}$.

Now, with $|\alpha| \leq m$, we take the $D^{\alpha}$ derivative of (3.6), multiply it by $D^{\alpha} \hat{\rho}$ and integrate it with respect to $x$. Similarly, we take the $D^{\alpha}$ derivative of (3.7), multiply it by $D^{\alpha} \varphi$ and integrate it with respect to $x$. We sum up the resulting identities. Using the notation

$$
\langle f, g\rangle=\int_{\Pi^{2}} f g d x
$$

we find:

$$
\begin{aligned}
0= & \left\langle D^{\alpha} \hat{\rho}, D^{\alpha} \hat{\rho}_{t}\right\rangle+\left\langle D^{\alpha} \varphi, D^{\alpha} \varphi_{t}\right\rangle \\
& +\left\langle D^{\alpha} \hat{\rho}, D^{\alpha}\left(\left(\Omega \cdot \nabla_{x}\right) \hat{\rho}\right)\right\rangle+c\left\langle D^{\alpha} \varphi, D^{\alpha}\left(\left(\Omega \cdot \nabla_{x}\right) \varphi\right)\right\rangle \\
& +\left\langle D^{\alpha} \hat{\rho}, D^{\alpha}\left(\lambda(\hat{\rho})\left(\Omega^{\perp} \cdot \nabla_{x}\right) \varphi\right)\right\rangle+\left\langle D^{\alpha} \varphi, D^{\alpha}\left(\lambda(\hat{\rho})\left(\Omega^{\perp} \cdot \nabla_{x}\right) \hat{\rho}\right)\right\rangle \\
& -\mu\left\langle D^{\alpha} \varphi, D^{\alpha} \Delta_{x} \varphi\right\rangle \\
& -\mu\left\langle D^{\alpha} \varphi, D^{\alpha}\left(\nabla_{x} h(\hat{\rho}) \cdot \nabla_{x} \varphi\right)\right\rangle \\
= & I_{1}+\ldots+I_{5} .
\end{aligned}
$$

Then:

$$
I_{1}=\frac{1}{2} \frac{d}{d t}\left(\left\|D^{\alpha} \hat{\rho}\right\|^{2}+\left\|D^{\alpha} \varphi\right\|^{2}\right)
$$

and

$$
I_{4}=\mu\left\|D^{\alpha} \nabla_{x} \varphi\right\|^{2},
$$

where $\|\cdot\|$ just indicates an $L^{2}$ norm. Now, for the remaining terms, we have the following lemma

Lemma 3.3. We have:

$$
\begin{aligned}
& \left|I_{k}\right| \leq C\left(\|\hat{\rho}\|_{W^{1, \infty}}+\|\varphi\|_{W^{1, \infty}}\right)\left(\|\hat{\rho}\|_{H^{m}}^{2}+\|\varphi\|_{H^{m}}^{2}\right), \quad k=2,3, \\
& \left|I_{5}\right| \leq \frac{\mu}{2}\left\|\nabla D^{\alpha} \varphi\right\|^{2}+C\left(\|\hat{\rho}\|_{W^{1, \infty}}^{2}+\|\varphi\|_{W^{1, \infty}}^{2}\right)\left(\|\hat{\rho}\|_{H^{m}}^{2}+\|\varphi\|_{H^{m}}^{2}\right),
\end{aligned}
$$

where $C$ denote generic constants depending on the parameters of the problem.

The proof of the lemma is postponed at the end. 
Adding all these terms together for all possible indices $\alpha$ such that $|\alpha| \leq m$, we have,

$$
\begin{aligned}
\frac{1}{2} \frac{d}{d t}\left(\|\hat{\rho}\|_{H^{m}}^{2}+\|\varphi\|_{H^{m}}^{2}\right)+\mu\left\|\nabla_{x} \varphi\right\|_{H^{m}}^{2} \leq \frac{\mu}{2}\left\|\nabla_{x} \varphi\right\|_{H^{m}}^{2}+ \\
+C\left(\|\hat{\rho}\|_{W^{1, \infty}}^{2}+\|\varphi\|_{W^{1, \infty}}^{2}+1\right)\left(\|\hat{\rho}\|_{H^{m}}^{2}+\|\varphi\|_{H^{m}}^{2}\right) .
\end{aligned}
$$

For $m \geq \frac{n}{2}+1$, we have

$$
\|\hat{\rho}\|_{W^{1, \infty}}+\|\varphi\|_{W^{1, \infty}} \leq C\left(\|\hat{\rho}\|_{H^{m}}+\|\varphi\|_{H^{m}}\right)
$$

and get

$$
\frac{1}{2} \frac{d}{d t}\left(\|\hat{\rho}\|_{H^{m}}^{2}+\|\varphi\|_{H^{m}}^{2}\right)+\frac{\mu}{2}\left\|\nabla_{x} \varphi\right\|_{H^{m}}^{2} \leq C\left(\|\hat{\rho}\|_{H^{m}}^{2}+\|\varphi\|_{H^{m}}^{2}+1\right)^{2} .
$$

Gronwall's inequality leads to the local existence of a solution $(\hat{\rho}, \varphi)$ in $L^{\infty}\left([0, T], H^{m}\left(\Pi^{2}\right)\right)$ which, if $\mu>0$, also belongs to $L^{2}\left([0, T], H^{m+1}\left(\Pi^{2}\right)\right)$ and which satisfies the a priori bound (3.8). To get time regularity, we directly use eqs. (3.6), (3.7), take the $H^{m-1}$ norm, apply Lemma 3.2 , and find

$$
\left\|\hat{\rho}_{t}\right\|_{H^{m-1}}+\left\|\varphi_{t}\right\|_{H^{m-1}} \leq C\|\varphi\|_{H^{m+1}}+C\left(\|\hat{\rho}\|_{W^{1, \infty}}+\|\varphi\|_{W^{1, \infty}}\right)\left(\|\hat{\rho}\|_{H^{m}}+\|\varphi\|_{H^{m}}\right) .
$$

Using the previous estimates, we deduce that $(\hat{\rho}, \varphi)$ also belongs to $H^{1}\left([0, T], H^{m-1}\left(\Pi^{2}\right)\right)$. The estimates on $\hat{\rho}$ immediately transfer to $\rho$ since $a(\rho)$ is smooth and invertible for $\rho>0$.

Proof of Lemma 3.3. Estimate of $I_{5}$ : Using Green's formula and Cauchy-Schwartz inequality, we have:

$$
\begin{aligned}
\left|I_{5}\right| & \leq \mu\left\|\nabla_{x} D^{\alpha} \varphi\right\|\left\|\nabla_{x} h(\hat{\rho}) \cdot \nabla_{x} \varphi\right\|_{H^{m-1}} \\
& \leq C\left\|\nabla_{x} D^{\alpha} \varphi\right\|\left(\|\hat{\rho}\|_{H^{m}}\left\|\nabla_{x} \varphi\right\|_{L^{\infty}}+\left\|\nabla_{x} \hat{\rho}\right\|_{L^{\infty}}\|\varphi\|_{H^{m}}\right) \\
& \leq \frac{\mu}{2}\left\|\nabla_{x} D^{\alpha} \varphi\right\|^{2}+C\left(\|\hat{\rho}\|_{H^{m}}\left\|\nabla_{x} \varphi\right\|_{L^{\infty}}+\left\|\nabla_{x} \hat{\rho}\right\|_{L^{\infty}}\|\varphi\|_{H^{m}}\right)^{2} \\
& \leq \frac{\mu}{2}\left\|\nabla_{x} D^{\alpha} \varphi\right\|^{2}+C\left(\|\hat{\rho}\|_{W^{1, \infty}}^{2}+\|\varphi\|_{W^{1, \infty}}^{2}\right)\left(\|\hat{\rho}\|_{H^{m}}^{2}+\|\varphi\|_{H^{m}}^{2}\right) .
\end{aligned}
$$

The second inequality uses Lemma 3.2 and the third one uses Young's inequality.

Estimate of $I_{3}$ : We write

$$
\begin{aligned}
I_{3}= & \left\langle D^{\alpha} \hat{\rho}, \lambda(\hat{\rho})\left(\Omega^{\perp} \cdot \nabla_{x}\right) D^{\alpha} \varphi\right\rangle+\left\langle D^{\alpha} \varphi,\left(\lambda(\hat{\rho})\left(\Omega^{\perp} \cdot \nabla_{x}\right) D^{\alpha} \hat{\rho}\right)\right\rangle \\
& +\left\langle D^{\alpha} \hat{\rho},\left(D^{\alpha}\left(\lambda(\hat{\rho})\left(\Omega^{\perp} \cdot \nabla_{x}\right) \varphi\right)-\lambda(\hat{\rho})\left(\Omega^{\perp} \cdot \nabla_{x}\right) D^{\alpha} \varphi\right)\right\rangle \\
& +\left\langle D^{\alpha} \varphi,\left(D^{\alpha}\left(\lambda(\hat{\rho})\left(\Omega^{\perp} \cdot \nabla_{x}\right) \hat{\rho}\right)-\lambda(\hat{\rho})\left(\Omega^{\perp} \cdot \nabla_{x}\right) D^{\alpha} \hat{\rho}\right)\right\rangle \\
= & J_{1}+J_{2}+J_{3} .
\end{aligned}
$$

Using Green's formula, we find

$$
\begin{aligned}
\left|J_{1}\right| & =\left|\left\langle\nabla_{x} \cdot\left(\lambda(\hat{\rho}) \Omega^{\perp}\right) D^{\alpha} \hat{\rho}, D^{\alpha} \varphi\right\rangle\right| \\
& \leq C\left(\|\hat{\rho}\|_{W^{1, \infty}}+\|\varphi\|_{W^{1, \infty}}\right)\left(\|\hat{\rho}\|_{H^{m}}^{2}+\|\varphi\|_{H^{m}}^{2}\right) .
\end{aligned}
$$

Now, using Cauchy-Schwartz inequality and applying Lemma 3.2, we find that $J_{2}$ and $J_{3}$ satisfy the same inequality.

Estimate of $I_{2}$ : The proof is similar as for $I_{3}$ and is omitted. 
3.2. Existence in $3 \mathrm{D}$ without viscosity. In this section, we investigate the local existence for the inviscid $\mathrm{SOH}$ problem in 3 dimensions:

$$
\begin{aligned}
& \partial_{t} \rho+\nabla_{x} \cdot(\rho \Omega)=0, \\
& \rho\left(\partial_{t} \Omega+c \Omega \cdot \nabla_{x} \Omega\right)+P_{\Omega^{\perp}} \nabla_{x}(p(\rho))=0,
\end{aligned}
$$

where the parameters and data have the same meaning as in Section 3.1. We consider the system in the domain $\Pi^{3}=[0,1]^{3}$ with periodic boundary conditions.

For this purpose, we use the spherical coordinates associated to a fixed Cartesian basis. In this basis, denoting by $\theta \in[0, \pi]$ the latitude and $\varphi \in[0,2 \pi]$ the longitude, we have

$$
\Omega=(\sin \theta \cos \varphi, \sin \theta \sin \varphi, \cos \theta)^{T},
$$

and we let $\Omega_{\theta}$ and $\Omega_{\varphi}$ be the derivatives of $\Omega$ with respect to $\theta$ and $\varphi$. We note that

$$
\left|\Omega_{\theta}\right|=1, \quad\left|\Omega_{\varphi}\right|=\sin \theta .
$$

We will use the formulas

$$
\begin{aligned}
& \nabla_{x} \cdot \Omega=\Omega_{\theta} \cdot \nabla_{x} \theta+\Omega_{\varphi} \cdot \nabla_{x} \varphi \\
& P_{\Omega^{\perp}} a=\left(\Omega_{\theta} \cdot a\right) \Omega_{\theta}+\frac{\left(\Omega_{\varphi} \cdot a\right)}{\sin ^{2} \theta} \Omega_{\varphi}, \\
& \left(\Omega \cdot \nabla_{x}\right) \Omega=\left(\left(\Omega \cdot \nabla_{x}\right) \theta\right) \Omega_{\theta}+\left(\left(\Omega \cdot \nabla_{x}\right) \varphi\right) \Omega_{\varphi}, \\
& \Omega_{t}=\Omega_{\theta} \theta_{t}+\Omega_{\varphi} \varphi_{t},
\end{aligned}
$$

where $a$ is an arbitrary vector.

Introduce $\hat{\rho}$ and $\lambda(\hat{\rho})$ as in (3.5). Then, system (3.9), (3.10) becomes:

$$
\begin{aligned}
& \hat{\rho}_{t}+\Omega \cdot \nabla_{x} \hat{\rho}+\lambda(\hat{\rho}) \nabla_{x} \cdot \Omega=0, \\
& \Omega_{t}+c\left(\Omega \cdot \nabla_{x}\right) \Omega+\lambda(\hat{\rho}) P_{\Omega^{\perp}} \nabla_{x} \hat{\rho}=0,
\end{aligned}
$$

or,

$$
\begin{aligned}
& \hat{\rho}_{t}+\Omega \cdot \nabla_{x} \hat{\rho}+\lambda(\hat{\rho})\left(\Omega_{\theta} \cdot \nabla_{x} \theta+\Omega_{\varphi} \cdot \nabla_{x} \varphi\right)=0, \\
& \theta_{t}+c\left(\Omega \cdot \nabla_{x}\right) \theta+\lambda(\hat{\rho}) \Omega_{\theta} \cdot \nabla_{x} \hat{\rho}=0, \\
& \sin ^{2} \theta \varphi_{t}+c \sin ^{2} \theta\left(\Omega \cdot \nabla_{x}\right) \varphi+\lambda(\hat{\rho}) \Omega_{\varphi} \cdot \nabla_{x} \hat{\rho}=0 .
\end{aligned}
$$

Introducing

$$
U=\left(\begin{array}{c}
\hat{\rho} \\
\theta \\
\varphi
\end{array}\right)
$$

this system is written

$$
A_{0}(U) U_{t}+A_{1}(U) U_{x}+A_{2}(U) U_{y}+A_{3}(U) U_{z}=0,
$$

in Cartesian coordinates $x=(x, y, z)$, where $A_{k}(U), k=0, \ldots, 4$ are all symmetric matrices and

$$
A_{0}=\left(\begin{array}{ccc}
1 & 0 & 0 \\
0 & 1 & 0 \\
0 & 0 & \sin ^{2} \theta
\end{array}\right)
$$


If $\sin \theta>0$, then this system is a symmetrizable hyperbolic system. We can apply proposition 2.1 p. 425 of [41] and the following theorem follows immediately:

THEOREM 3.4. We assume that the initial data $\left(\rho_{0}, \theta_{0}, \varphi_{0}\right)$ belong to $H^{m}\left(\Pi^{3}\right)$ with $m>5 / 2$ with $\rho_{0}>0, \sin \theta_{0}>0$. Then, there exists a time $T>0$ and a unique solution $(\rho, \theta, \varphi)$ of the system (3.9)-(3.10) in $L^{\infty}\left([0, T], H^{m}\left(\Pi^{3}\right)\right) \cap H^{1}\left([0, T], H^{m-1}\left(\Pi^{3}\right)\right)$ such that $\rho$ remains positive.

REMARK 3.1. We see from (3.14) that in 3D, the symmetrizer degenerates at $\sin \theta=0$. So the structure condition $\sin \theta \geq C>0$ is needed. In the inviscid case, if the initial condition satisfies this condition, the solution itself satisfies it over a certain time interval by finite speed of propagation. However, if viscosity is added, the speed of propagation becomes infinite and this structure condition is instantaneously lost. This is why we restrict ourselves to the inviscid case in $3 D$.

4. Conclusion. In this paper, we have derived hydrodynamic systems from kinetic models of self-propelled particles with alignment interaction and attractionrepulsion force. We have particularly focused on the inclusion of diffusion terms under the assumption of weakly non-local interactions. Then, we have proved the local-in-time existence of solutions for the viscous system in 2D and a similar result for the inviscid system in 3D. The methods rely on a suitable symmetrization and on the energy method. Adding an attraction-repulsion potential has greatly extended the work in [13]. The weakly nonlocal scaling allows us to derive new macroscopic effects such as viscosity and capillary (or Korteweg) forces. This work also provides rigorous existence results on the macroscopic models whereas [13] contains only formal results. Future works in this direction will consist in continuing the exploration of the mathematical structure of the system and particularly, trying to prove local existence of the viscous system in $3 \mathrm{D}$ and the treatment of the geometric singularity near $\sin \theta=0$. Another direction of work will consist of the numerical quantification of the viscosity as a consequence of the non-locality of the interaction.

\section{REFERENCES}

[1] M. Aldana And C. Huepe, Phase transitions in self-driven many-particle systems and related non-equilibrium models: a network approach, J. Stat. Phys., 112 (2003), pp. 135-153.

[2] I. AoKI, A simulation study on the schooling mechanism in fish, Bulletin of the Japan Society of Scientific Fisheries, 48 (1982), pp. 1081-1088.

[3] F. Bolley, J. A. Cañizo, and J. A. Carrillo, Mean-field limit for the stochastic Vicsek model, Appl. Math. Lett., 25 (2011), pp. 339-343.

[4] F. Bouchut, On zero pressure gas dynamics, in Advances in kinetic theory and computing, Series on Advances in Mathematics for Applied Sciences, Vol 22, World Scientific, 1994, pp. 171-190.

[5] J. A. Carrillo, M. R. D’Orsogna, and V. Panferov, Double milling in self-propelled swarms from kinetic theory, Kinetic and Related Models 2 (2009), pp. 363-378.

[6] J. A. Carrillo, A. Klar, S. Martin, and S. Tiwari, Self-propelled interacting particle systems with roosting force, Math. Models Methods Appl. Sci., 20 (2010), pp. 1533-1552.

[7] H. Chaté, F. Ginelli, G. Grégoire, and F. Raynaud, Collective motion of self-propelled particles interacting without cohesion, Phys. Rev. E, 77 (2008), pp. 046113.

[8] Y-L. Chuang, M. R. D’Orsogna, D. Marthaler, A. L. Bertozzi, and L. S. Chayes, State transitions and the continuum limit for a $2 D$ interacting, self-propelled particle system, Physica D, 232 (2007), pp. 33-47.

[9] I. D. Couzin, J. Krause, R. James, G. D. Ruxton, and N. R. Franks, Collective memory and spatial sorting in animal groups, J. theor. Biol., 218 (2002), pp. 1-11.

[10] A. Czirók, E. Ben-Jacob, I. Cohen, and T. Vicsek, Formation of complex bacterial colonies via self-generated vortices, Phys. Rev E , 54 (1996), pp. 1791-1801. 
[11] P. Degond, A. Frouvelle, And J. G. Liu, Macroscopic limits and phase transition in a system of self-propelled particles, Journal of Nonlinear Science, 23 (2013), pp. 427-456.

[12] P. Degond And J. HuA, Self-Organized Hydrodynamics with congestion and path formation in crowds, preprint arXiv:1207.3522, 2012.

[13] P. Degond And S. Motsch, Continuum limit of self-driven particles with orientation interaction, Math. Models Methods Appl. Sci., 18, Suppl. (2008), pp. 1193-1215.

[14] P. Degond And S. Motsch, A macroscopic model for a system of swarming agents using curvature control, J. Stat. Phys., 143 (2011), pp. 685-714.

[15] P. Degond, T. YAng, Diffusion in a continuum model of self-propelled particles with alignment interaction, Math. Models Methods Appl. Sci., 20 Suppl. (2010), pp. 1459-1490.

[16] B. Després, F. Lagoutière, And N. Seguin, Weak solutions to Friedrichs systems with convex constraints, Nonlinearity, 24 (2011), pp. 3055-3081.

[17] M. Doi And S. F. Edwards, The theory of polymer dynamics, Clarendon Press, 1999.

[18] M. R. D'Orsogna, Y. L. Chuang, A. L. Bertozzi, and L. Chayes, Self-propelled particles with soft-core interactions: patterns, stability and collapse, Phys. Rev. Lett., 96 (2006), p. 104302.

[19] A. Frouvelle, A continuous model for alignment of self-propelled particles with anisotropy and density-dependent parameters, Math. Models Methods Appl. Sci., 22 (2012), p. 1250011.

[20] A. Frouvelle And J. G. Liu, Dynamics in a kinetic model of oriented particles with phase transition, SIAM J. Math. Anal., 44 (2012), pp. 791-826.

[21] G. Grégoire and H. Chaté, Onset of collective and cohesive motion, Phys. Rev. Lett., 92 (2004), p. 025702.

[22] G. Grégoire, H. Chaté, And Y. Tu, Moving and staying together without a leader, Physica D, 181 (2003), pp. 157-170.

[23] D. J. Konteweg, Sur la forme que prennent les équations du mouvement des fluides si l'on tient compte des forces capillares causées par les variations de densité, Arch. Néerlandaises des Sci. Exact et Natur. Sér. II, 6 (1901), pp. 1-24.

[24] V. L. Kulinskit, V. I. Ratushnaya, A. V. Zvelindovsky, and D. Bedeaux, Hydrodynamic model for a system of self-propelling particles with conservative kinematic constraints, Europhys. Lett., 71 (2005), pp. 207-213.

[25] F.-H. LIN AND C. LiU, Existence of solutions for the Ericksen-Leslie system, Arch. Rational Mech. Anal., 154 (2000), pp. 135-156.

[26] F.-H. Lin, J. Lin, AND C. WANG, Liquid crystal flows in two-dimensions, Arch. Rational Mech. Anal., 197 (2010), pp. 297-336.

[27] A. Mogilner And L. Edelstein-Keshet, A non-local model for a swarm, J. Math. Biol., 38 (1999), pp. 534-570

[28] A. Mogilner, L. Edelstein-Keshet, L. Bent, And A. Spiros, Mutual interactions, potentials, and individual distance in a social aggregation, J. Math. Biol., 47 (2003), pp. 353-389.

[29] S. Motsch And L. NAvoret, Numerical simulations of a non-conservative hyperbolic system with geometric constraints describing swarming behavior, Multiscale Model. Simul., 9 (2011), pp. 1253-1275.

[30] L. ONSAGER, The effects of shape on the interaction of colloidal particles, Annals of the New York Academy of Sciences, 51 (1949), pp. 627-659.

[31] J. K Parrish, S. V. Viscido, And D. Grunbaum, Self-organized fish schools: an examination of emergent properties, Biological Bulletin, Marine Biological Laboratory, Woods Hole (2002), pp. 296-305.

[32] V. I. Ratushnaya, D. Bedeaux, V. L. Kulinskit, and A. V. Zvelindovsky, Collective behaviour of self propelling particles with kinematic constraints ; the relations between the discrete and the continuous description, Physica A, 381 (2007), pp. 39-46.

[33] V. I. Ratushnaya, V. L. Kulinskit, A. V. Zvelindovsky, and D. Bedeaux, Hydrodynamic model for the system of self propelling particles with conservative kinematic constraints; two dimensional stationary solutions, Physica A, 366 (2006), pp. 107-114.

[34] J. RAUCH, Boundary value problems as limits of problems in all space, séminaire GoulaouicSchwartz (1978/1979), Ecole Polytechnique, Palaiseau, 1979.

[35] C. W. Reynolds, Flocks, herds and schools: a distributed behavioral model, Comput. Graph., 21 (1987), pp. 25-34.

[36] A.-S. Sznitman, Topics in propagation of chaos, École d'été de probabilités de Saint-Flour XIX-1989, Lecture Notes in Math, 1464 (1989), pp. 165-251.

[37] C. M. Topaz And A. L. BertozzI, Swarming patterns in a two-dimensional kinematic model for biological groups, SIAM J. Appl. Math, 65 (2004), pp. 152-174.

[38] C. M. Topaz, A. L. Bertozzi, and M. A. Lewis, A nonlocal continuum model for biological aggregation, Bull. Math. Biol., 68 (2006), pp. 1601-1623. 
[39] J. D. VAn DER WAALS, On the continuity of the gaseous and liquid states, Studies in Statistical Mechanics, vol. XIV, North-Holland, Amsterdam, 1988 (translation of the Leiden thesis of J. D. Van Der Waals, dated 1873).

[40] T. Vicsek, A. Czirók, E. Ben-Jacob, I. Cohen, and O. Shochet, Novel type of phase transition in a system of self-driven particles, Phys. Rev. Lett., 75 (1995), pp. 1226-1229.

[41] M. E. TAYLOR, Partial Differential Equations III, Applied Mathematical Sciences Series Vol. 117, Springer, 1996, 2011.

[42] C. Villani, Topics in optimal transportation, AMS Graduate Studies in Mathematics, Vol. 58, AMS, Providence, 2003. 